\title{
ON ALGEBRAIC EQUIVALENCE BETWEEN PAIRS OF LINEAR TRANSFORMATIONS ${ }^{1}$ ) \\ BY \\ URI FIXMAN
}

Introduction. In [1] the authors studied pairs $\mathscr{A}, \mathscr{B}$ of linear transformations of a complex vector space $V$ into a complex vector space $W(V, W$ possibly of infinite dimension). The original motivation for this study was its application to a perturbation theory of differential eigenvalue problems developed by Aronszajn (see the introduction to the cited paper). Our aim here is to make a small contribution to the algebraic problem of equivalence of pairs of linear transformations. The pair $\mathscr{A}, \mathscr{B}: V \rightarrow W$ is said to be equivalent (or isomorphic) to the pair $\mathscr{A}^{\prime}, \mathscr{B}^{\prime}: V^{\prime} \rightarrow W^{\prime}$ in case there exist linear isomorphisms $\phi$ of $V$ onto $V^{\prime}$ and $\psi$ of $W$ onto $W^{\prime}$ such that $\mathscr{A}^{\prime} \phi=\psi \mathscr{A}, \mathscr{B} \mathscr{B}^{\prime} \phi=\psi \mathscr{B}$. Our considerations will be valid for vector spaces over any algebraically closed field; however we prefer to adhere to the terminology suitable for complex vector spaces.

For finite-dimensional vector spaces the problem was solved by Kronecker who found canonical forms for pencils of rectangular matrices. In addition to [1] and the brief statement 1.7 below, one may find an exposition of Kronecker's result akin to our approach in [5] (which contains further references). In the so-called nonsingular case, in which an invertible linear combination of $\mathscr{A}$ and $\mathscr{B}$ exists (and is known), the problem reduces to that of similarity of single operators. It well known that it may then be treated as the isomorphism problem for unitary modules over the complex polynomials, for which a vast literature exists. It appears that the infinite-dimensional general case was not considered before [1].

To treat the general case it is technically convenient to replace the structure $(\mathscr{A}, \mathscr{B} ; V, W)$ by the following simple analog to a module. Let $\mathscr{T}$ denote the 2-dimensional vector space of pairs of complex numbers. A (linear) system $(V, W)$ is a pair of complex vector spaces $V, W$ together with a system operation which assigns to every pair of elements $A \in \mathscr{T}, v \in V$ an element $A v$ of $W$ so that

(i) for every $A \in \mathscr{T}$ the map $v \rightarrow A v$ is a linear transformation of $V$ into $W$;

(ii) $(\alpha A+\beta B) v=\alpha(A v)+\beta(B v)$ for $v \in V, A, B \in \mathscr{T}$ and $\alpha, \beta$ complex.

Otherwise expressed, the map $(A, v) \rightarrow A v$ is a bilinear transformation of

Presented to the Society, August 31, 1960 under the title of On torsion-free linear sysetms; received by the editors June 14, 1962.

(1) Supported in part by the Office of Ordnance Research under Contract DA-19-020ORD-4912 with Yale University. 
$\mathscr{T} \times V$ into $W$. To $(\mathscr{A}, \mathscr{B} ; V, W)$ we attach the system $(V, W)$ with the system operation defined by $(\alpha, \beta) v=(\alpha \mathscr{A}+\beta \mathscr{B}) v$. We carry the discussion through in terms of systems; the results obtained can of course be reinterpreted in terms of the original transformations. Although we do not have here a complete analog to the ring of coefficients of a module (in the nonsingular case $V=W, \mathscr{A}=$ identity, the elements of $\mathscr{T}$ correspond just to the linear polynomials in the ring of the module associated with $\mathscr{B}$ ), it is possible to extend module-theoretic concepts to systems. It is in this spirit that the undefined terms in the introduction may be understood. Since previous familiarity with [1] is not assumed, we state in $\S 1$ the definitions and results of [1] which will be needed in the sequel.

One of the main concepts of [1] was an analog to the notion of a pure submodule. A subsystem of a system $(V, W)$ is quasi-spectral in $(V, W)$ if it is a direct summand in every finite-dimensional extension contained in $(V, W)$. A given system $(V, W)$ was investigated in terms of quasi-spectral direct sums of quasispectrally-irreducible (q.s.irr.) subsystems. This was carried out for all q.s.irr. subsystems which are of finite dimension or of torsion type. The above direct sums were shown to correspond to linearly independent subsets of certain auxiliary vector spaces, the dimensions of which yielded isomorphism invariants. These invariants may be regarded as the multiplicities of appearance of q.s.irr. types in $(V, W)$.

In the first half of the present work we study a new family of q.s.irr. types: In $\$ 2$ we introduce (torsion-free) rank for systems; while in $\$ 3$ we characterize the isomorphism types of torsion-free systems of rank 1-these are q.s.irr.-by classes of "height functions" and find their algebras of endomorphisms. These results run parallel to the corresponding ones in the theory of abelian groups (see [2] and [3]). Because we include the singular case, there exist q.s.irr. torsionfree systems of rank higher than 1.

In the rest of the paper we formulate conditions for a family of isomorphic q.s.irr. subsystems to have a quasi-spectral direct sum ( $\$ 4)$, and apply them to torsion-free subsystems of rank 1 ( $\$ 5)$. The case of subsystems which are not isomorphic is also briefly dealt with. Here the mentioned linearly independent subsets of vector spaces have to be replaced by independent subsets of modules of homomorphisms modulo certain subsets. Only for a very restricted class of torsion-free systems of rank 1 do these subsets always form submodules, thus enabling us to attach to $(V, W)$ corresponding multiplicity invariants (cf. the discussion towards the end of $\S 4$ and Theorems 5.12, 5.13).

1. Preliminaries. A system $\left(V^{\prime}, W^{\prime}\right)$ is called a subsystem of the system $(V, W)$ if and only if $V^{\prime} \subseteq V, W^{\prime} \subseteq W$ and the system operation of $\left(V^{\prime}, W^{\prime}\right)$ is the restriction of the system operation of $(V, W)$ to $\mathscr{T} \times V^{\prime}$ (implying that the range $\mathscr{T} V^{\prime}=\left\{\sum A_{i} v_{i}^{\prime} \mid A_{i} \in \mathscr{T}, v_{i}^{\prime} \in V^{\prime}\right\}$ is contained in $W^{\prime}$. The space $W^{\prime}$ is termed the range space of $\left.\left(V^{\prime}, W^{\prime}\right)\right)$. The subsystems of a system $(V, W)$ form a 
complete modular lattice under the operations of taking sums $\Sigma\left(V_{i}, W_{i}\right)$ $=\left(\Sigma V_{i}, \Sigma W_{i}\right)$ and intersections $\bigcap\left(V_{i}, W_{i}\right)=\left(\bigcap V_{i}, \bigcap W_{i}\right)$ (with the induced system operations) or equivalently under the partial order: $\left(V^{\prime}, W^{\prime}\right) \subseteq\left(V^{\prime \prime}, W^{\prime \prime}\right)$ if and only if $V^{\prime} \subseteq V^{\prime \prime}$ and $W^{\prime} \subseteq W^{\prime \prime}$.

The meaning of the derived notions independent set of subsystems, (discrete) direct sum of subsystems (denoted by $\Sigma+\left(V_{i}, W,\right)$ ) and decomposable system is now obvious. It will be convenient to say that a subsystem $\left(V^{\prime}, W^{\prime}\right)$ of $(V, W)$ is spectral in $(V, W)$ in case it is a direct summand of $(V, W)$; i.e., when there exists a subsystem $\left(V^{\prime \prime}, W^{\prime \prime}\right)$ such that $(V, W)=\left(V^{\prime}, W^{\prime}\right)+\left(V^{\prime \prime}, W^{\prime \prime}\right)$. A system $(V, W)$ is of finite dimension, if and only if both $V$ and $W$ are finite-dimensional vector spaces. We then define $\operatorname{dim}(V, W)=\operatorname{dim} V+\operatorname{dim} W$ (this is the ordinary lattice theoretic dimension). The convention that an empty sum of subsystems is the null subsystem $(0,0)$ will be followed. The above definition of partial order will be used also for pairs of subsets $(M, N)$ where $M \subseteq V, N \subseteq W$ which do not necessarily constitute subsystems. If $(M, N) \subseteq(V, W)$ where $(V, W)$ is a system, the subsystem spanned by $(M, N)$, i.e., the least subsystem of $(V, W)$ which contains $(M, N)$, will be denoted by $[M, N]$ (while $[M]$ and $[N]$ denote spanned vector spaces).

A homomorphism of a system $(V, W)$ into a system $\left(V^{\prime}, W^{\prime}\right)$ is a pair $(\phi, \psi)$ of linear transformations of $V$ into $V^{\prime}$ and $W$ into $W^{\prime}$ respectively such that $A \phi v=\psi A v$ for every $A \in \mathscr{T}, v \in V$. The terms isomorphism, isomorphic systems and endomorphism are now self-exlanatory. If $(\phi, \psi)$ is a homomorphism of $(V, W)$ into $\left(V^{\prime}, W^{\prime}\right)$ its image $(\phi, \psi)(V, W)=(\phi V, \psi W)$ is a subsystem of $\left(V^{\prime}, W^{\prime}\right)$, and its kernel $\operatorname{Ker}(\phi, \psi)=(\operatorname{Ker} \phi, \operatorname{Ker} \psi)$ is a subsystem of $(V, W)$. If $\operatorname{Ker}(\phi, \psi)=(0,0),(\phi, \psi)$ is called a monomorphism. If $\left(V^{\prime}, W^{\prime}\right)$ is a subsystem of $(V, W)$, the quotient system $(V, W) /\left(V^{\prime}, W^{\prime}\right)$ is the pair $\left(V / V^{\prime}, W / W^{\prime}\right)$ with the system operation $A\left(v+V^{\prime}\right)=A v+W^{\prime}, A \in \mathscr{T}, v \in V$. The usual homomorphism and isomorphism theorems hold.

The set $\operatorname{Hom}\left(V, W ; V^{\prime}, W^{\prime}\right)$ of homomorphisms of $(V, W)$ into $\left(V^{\prime}, W^{\prime}\right)$, with the operations $\left(\phi_{1}, \psi_{1}\right)+\left(\phi_{2}, \psi_{2}\right)=\left(\phi_{1}+\phi_{2}, \psi_{1}+\psi_{2}\right)$ and $\alpha(\phi, \psi)=(\alpha \phi, \alpha \psi)$ ( $\alpha$ complex) is a vector space. No confusion with a null system will arise by denoting the zero of $\operatorname{Hom}\left(V, W ; V^{\prime}, W^{\prime}\right)$ by $(0,0)$. The set $\operatorname{End}(V, W)=\operatorname{Hom}(V, W ; V, W)$ can be made into an algebra over the complex numbers with the identity element $(1,1)$ by adding the operation of multiplication $\left(\phi_{1}, \psi_{1}\right)\left(\phi_{2}, \psi_{2}\right)=\left(\phi_{1} \phi_{2}, \psi_{1} \psi_{2}\right)$. Idempotents of $\operatorname{End}(V, W)$ are called projections and are related to direct sums in the usual manner.

A system $(V, W)$ is called nonsingular if and only if there exists an element $A$ in $\mathscr{T}$ such that the linear transformation $\phi: v \rightarrow A v$ is an isomorphism of $V$ onto $W$. In this case $\left(1, \phi^{-1}\right)$ is an isomorphism of $(V, W)$ onto the system $(V, V)$ with the system operation $\circ$ defined by $B \circ v=\phi^{-1} B v, B \in \mathscr{T}, v \in V$. In the latter system the elements of $\mathscr{T}$ induce linear operators of $V$ into itself, $A$ induces the identity, and the operator $\mathscr{C}$ induced by any element independent of $A$ (to- 
gether with the knowledge of this element) determines the system. The invariant subspaces of $\mathscr{C}$ correspond in a 1-1 fashion to the subsystems $\left(V^{\prime}, W^{\prime}\right)$ of $(V, W)$ such that $\mathscr{T} V^{\prime}=W^{\prime}$. Thus the concept of a nonsingular system is essentially equivalent to that of a single operator $\mathscr{C}$ of a vector space $V$ and therefore also to the module over the complex polynomials defined by $p(\zeta) v=p(\mathscr{C}) v, p(\zeta)$ a polynomial, $v \in V$.

A subsystem $\left(V^{\prime}, W^{\prime}\right)$ of a system $(V, W)$ is said to be quasi-spectral (q.s.) in $(V, W)$ if and only if it is spectral in every subsystem $\left(V^{\prime \prime}, W^{\prime \prime}\right)$ such that $\left(V^{\prime}, W^{\prime}\right) \subseteq\left(V^{\prime \prime}, W^{\prime \prime}\right) \subseteq(V, W)$ and $\left(V^{\prime \prime}, W^{\prime \prime}\right) /\left(V^{\prime}, W^{\prime}\right)$ is of finite dimension. A system is quasi-spectrally-irreducible (q.s.irr.) if it is not a null system and does not contain nontrivial q.s. subsystems. By modularity it follows that every spectral subsystem of $(V, W)$ is q.s. in $(V, W)$. Hence a finite-dimensional system is q.s.irr. if and only if it is indecomposable. We call a system $(V, W)$ algebraically compact in case it is spectral in every system which contains $(V, W)$ as a q.s. subsystem. The following properties are taken from [1].

1.1. If $\left(V^{\prime \prime}, W^{\prime \prime}\right)$ is q.s. in $\left(V^{\prime}, W^{\prime}\right)$ and $\left(V^{\prime}, W^{\prime}\right)$ is q.s. in $(V, W)$, then $\left(V^{\prime \prime}, W^{\prime \prime}\right)$ is q.s. in $(V, W)$.

This together with an obvious converse imply that a subsystem of $(V, W)$ is minimal among the non-null q.s. subsystems of $(V, W)$ if and only if it is a q.s. subsystem which is q.s.irr.

1.2. If $\left\{\left(V_{1}, W_{i}\right)\right\}$ is a family of q.s. subsystems of $(V, W)$ which is a directed set under inclusion, then $\sum_{i}\left(V_{i}, W_{i}\right)$ is q.s. in $(V, W)$.

1.3. If $\left(V^{\prime \prime}, W^{\prime \prime}\right) \subseteq\left(V^{\prime}, W^{\prime}\right) \subseteq(V, W)$ and $\left(V^{\prime}, W^{\prime}\right)$ is q.s. in $(V, W)$, then $\left(V^{\prime}, W^{\prime}\right) /\left(V^{\prime \prime}, W^{\prime \prime}\right)$ is q.s. in $(V, W) /\left(V^{\prime \prime}, W^{\prime \prime}\right)$. Conversely, if $\left(V^{\prime}, W^{\prime}\right) /\left(V^{\prime \prime}, W^{\prime \prime}\right)$ is q.s. in $(V, W) /\left(V^{\prime \prime}, W^{\prime \prime}\right)$ and in addition $\left(V^{\prime \prime}, W^{\prime \prime}\right)$ is q.s. in $(V, W)$, then $\left(V^{\prime}, W^{\prime}\right)$ is q.s. in $(V, W)$.

1.4. If $(X, Y),\left\{\left(V_{i}, W_{i}\right)\right\}$ and $\left\{\left(X_{i}, Y_{i}\right)\right\}$ are subsystems of $(V, W)$ such that $\left(V_{i}, W_{i}\right)=(X, Y)+\left(X_{i}, Y_{i}\right)$ for every $i$, then $(V, W)=(X, Y)+\Sigma+\left(X_{i}, Y_{i}\right)$ if and only if $(V, W) /(X, Y)=\Sigma+\left(\left(V_{i}, W_{i}\right) /(X, Y)\right)$.

1.5. Finite-dimensional systems and divisible systems $((V, W)$ is divisible if $A V=W$ for every $0 \neq A \in \mathscr{T})$ are algebraically compact. Unlike divisible modules over principal ideal-rings, divisible subsystems are not necessarily spectral.

To describe particular systems we need the following notation. Let $A, B$ be an (ordered) basis of $\mathscr{T}$. We make a 1-1 correspondence between representatives of the 1-dimensional subspaces of $\mathscr{T}$ and the extended complex plane $\Theta$ by defining $B_{\theta}=B-\theta A, \theta$ a finite complex number, $B_{\infty}=A$. This parametrization depends on the basis in the following manner: if $C=\alpha A+\beta B, D=\gamma A+\delta B$ is another basis, then $D_{\theta^{\prime}}$ and $B_{\theta}$ are proportional if and only if $\theta^{\prime}$ is related to $\theta$ by the Moebius transformation $\theta^{\prime}=(\delta \theta+\gamma) /(\beta \theta+\alpha)$. Whenever such a parametrization is used it is tacitly assumed that a fixed basis $A, B$ of $\mathscr{T}$ has been chosen. For a given system $(V, W)$ and $C \in \mathscr{T}$ denote $N(C)=\{v \in V \mid C v=0\}$. 
A nonzero element of $N\left(B_{\theta}\right)$ is called an eigenvector corresponding to the eigenvalue $\theta$. A system $(V, W)$ with $\operatorname{dim} V=m+1, m=0,1,2, \cdots$, is said to be of type

$I_{m}:$ if and only if $\operatorname{dim} W=m$ and $\operatorname{dim} N(C)=1$ for every $0 \neq C \in \mathscr{T}$.

$\mathrm{II}_{m}^{\boldsymbol{\theta}}$ : if and only if $\operatorname{dim} W=m+1, \operatorname{dim} N\left(B_{\theta}\right)=1$ and $N\left(B_{\eta}\right)=0$ for $\theta \neq \eta \in \Theta$.

III $_{m}$ : if and only if $\operatorname{dim} W=m+2$ and $N(C)=0$ for every $0 \neq C \in \mathscr{T}$. In this case we allow $m=-1$.

We may further describe such systems $(V, W)$ as follows. Let $A, B$ be a basis of $\mathscr{T}$, and let $p \leqq r \leqq s \leqq q$ be integers or the symbols $-\infty, \infty$. We denote by $C_{r}^{p}{ }_{s}^{q}(A, B ; V, W)$ the set of all sequences $\left\{v_{k}\right\}_{p}^{q}$ with elements in $V$ which satisfy the relations $A v_{k}=B v_{k-1}$ for every $k$ such that $p \leqq k-1 \leqq k \leqq q$ and $v_{k}=0$ for $p \leqq k<r$ and $s<k \leqq q$. Such a sequence is called a chain with range elements $w_{k}=A v_{k}$ (if $q<\infty$, denote $\left.w_{q+1}=B v_{q}\right)$. A proper chain in $C_{r}^{p q}(A, B ; V, W)$ is a chain in this set such that the sets $\left\{v_{k}\right\}_{r}^{s}$ and

$\left\{w_{k} \mid k \geqq r+1\right.$ if $p<r, k \geqq r$ if $p=r, k \leqq s$ if $s<q, k \leqq s+1$ if $\left.s=q\right\}$ are separately linearly independent. By the subsystem spanned by a chain $\left\{v_{k}\right\}$ we mean $\left[\left\{v_{k}\right\}, \varnothing\right]$.

1.6. A system $(V, W)$ is of type $\mathrm{I}_{m}, \mathrm{II}_{m}^{\theta}$ or $\mathrm{III}_{m}$ (here $m \geqq 0$ ) if and only if it is spanned by a proper chain in $C_{0}^{-\infty}{ }_{m}^{\infty}(A, B ; V, W), C_{0}^{0}{ }_{m}^{\infty}\left(A, B_{\theta} ; V, W\right)$ (here $A, B_{\infty}$ stands for the ordered basis $\left.B, A\right)$ or $C_{0}^{0}{ }_{m}^{m}(A, B ; V, W)$ respectively.

Hence the above types define unique isomorphism types (which are obviously distinct). As follows from the former characterizations of these types, the types $\mathrm{I}_{m}, \mathrm{III}_{m}$ do not depend on the choice of the basis $A, B$, while $\mathrm{II}^{\theta_{m}}$ depends on the proportionality class of $B_{\theta}$ only.

1.7. A finite-dimensional system is indecomposable if and only if it is of one of the types $\mathrm{I}_{m}, \mathrm{II}_{m}^{\theta}$ or $\mathrm{III}_{m}$.

Since every finite-dimensional system is obviously a direct sum of indecomposable ones, this together with 1.4 imply

1.8. A subsystem of a given system is q.s. if (and only if) it is spectral in every extension contained in the given system by a system of one of the types $I_{m}$, $\mathrm{II}_{m}^{\theta}$, or $\mathrm{III}_{m}$.

The following infinite-dimensional isomorphism types are q.s.irr. and divisible. A system $(V, W)$ is said to be of type $\mathrm{II}_{\infty}^{\theta}$ if it is spanned by a proper chain in $C_{-\infty}^{-\infty}{ }_{0}^{\infty}\left(A, B_{\theta} ; V, W\right)$ (see the above convention for $\left.\theta=\infty\right)$. Let $R$ denote the vector space of all the rational functions in an indeterminate $\zeta$ over the field of complex numbers, and let $A, B$ be a basis of $\mathscr{T}$. The systems $(R, R)_{A, B}$ where the system operation is defined by $((\alpha A+\beta B) f)(\zeta)=(\alpha+\beta \zeta) f(\zeta)$ have a common isomorphism type, independent of the basis $A, B$, which will be denoted by $\mathscr{R}$. For a more abstract characterization of these isomorphism types see [1]. 
A system is said to be torsion-free if it has no eigenvectors. Among the subsystems $(X, Y)$ of a system $(V, W)$ such that $(V, W) /(X, Y)$ is torsion-free there exists a (unique) least subsystem which is called the torsion part of $(V, W)$ and is denoted by $t(V, W)$. A system $(V, W)$ is called a torsion system in case $t(V, W)=(V, W)$ (in [1] the terms eigenvalue-free, eigenvalue part and eigenvalue systems were used).

1.9. $t(V, W)$ is a torsion system (cf. 2.1 below).

1.10. If $(X, Y) \subseteq t(V, W)$, then $t((V, W) /(X, Y))=t(V, W) /(X, Y)$. It follows that a homomorphic image of a torsion system is a torsion system (cf. 2.1).

1.11. $t(V, W)$ is the sum of all the subsystems of $(V, W)$ which are of the types $\mathrm{II}_{m}^{\theta}$ or $\mathrm{I}_{0}$. In particular, if $(V, W)$ is both torsion-free and a torsion system, then $(V, W)=(0,0)$.

1.12. $t(V, W)$ is the largest torsion subsystem of $(V, W)$. This follows from 1.9 and 1.11 .

1.13. $t(V, W)$ is q.s. in $(V, W)$. In more detail, there exists a transfinite increasing sequence $\left\{\left(V_{\alpha}, W_{\alpha}\right) \mid 0 \leqq \alpha \leqq \lambda\right\}$ of q.s. subsystems of $(V, W)$ such that: $\left(V_{0}, W_{0}\right)=(0,0) ; \quad\left(V_{\lambda}, W_{\lambda}\right)=t(V, W) ;$ if $\alpha \leqq \lambda$ is a limit ordinal, then $\left(V_{\alpha}, W_{\alpha}\right)=\bigcup_{\beta<\alpha}\left(V_{\beta}, W_{\beta}\right)$; if $\alpha \leqq \lambda$ has an immediate predecessor $\alpha-1$, then $\left(V_{\alpha}, W_{\alpha}\right) /\left(V_{\alpha-1}, W_{\alpha-1}\right)$ is one of the types $\mathrm{I}_{m}, \mathrm{II}_{m}^{\theta}$ or $\mathrm{II}_{\infty}^{\theta}$.

2. Rank. A subsystem $(X, Y)$ of a system $(V, W)$ is said to be torsion-closed (in $(V, W))$ if and only if $(V, W) /(X, Y)$ is torsion-free. It is immediately verified that the intersection of a family of torsion-closed subsystems of $(V, W)$ is torsionclosed in $(V, W)$. Hence if $(M, N)$ is a pair of subsets of $V, W$ respectively, there exists a least torsion-closed subsystem of $(V, W)$ which includes $(M, N)$. We call this subsystem the subsystem generated by $(M, N)$ in $(V, W)$, and denote it by $\operatorname{cl}(M, N)$ (or $\operatorname{cl}_{(V, W)}(M, N)$, when a qualification is necessary). The subsystems $\operatorname{cl}(\varnothing, N), \operatorname{cl}(\varnothing,\{w\})$ are said to be generated by $N, w$ respectively.

Note the different usage of the terms "generated" and "spanned."

2.1. LEMMA. (a) The operation $\mathrm{cl}$ is a closure operation; i.e., $\operatorname{cl}(M, N) \supseteq(M, N),\left(M_{1}, N_{1}\right) \supseteq\left(M_{2}, N_{2}\right)$ implies $\operatorname{cl}\left(M_{1}, N_{1}\right) \supseteq \operatorname{cl}\left(M_{2}, N_{2}\right)$ and $\operatorname{cl} \operatorname{cl}(M, N)=\operatorname{cl}(M, N)$.

(b) We have $\operatorname{cl}(M, N)=(M, N)$ if and only if $(M, N)$ constitutes a torsionclosed subsystem of $(V, W)$.

(c) $t(V, W)=\operatorname{cl}(\varnothing, \varnothing)$.

(d) The subsystem $\operatorname{cl}(M, N)$ is characterized by the relation $\operatorname{cl}(M, N) /[M, N]$ $=t((V, W) /[M, N])$.

(e) If the pair of subsets $(M, N)$ is included in the subsystem $(X, Y)$ of $(V, W)$, then $\operatorname{cl}_{(X, Y)}(M, N) \subseteq \mathrm{cl}_{(V, W)}(M, N)$. If in addition $(X, Y)$ is torsion-closed in $(V, W)$, then $\operatorname{cl}_{(X, Y)}(M, N)=\operatorname{cl}_{(V, W)}(M, N)$.

(f) If $(X, Y)$ is a subsystem of $(V, W)$ contained in $\operatorname{cl}(M, N)$ and $(\phi, \psi)$ is the natural homomorphism of $(V, W)$ onto $(V, W) /(X, Y)$, then 


$$
\mathrm{ci}_{(V, W) /(X, Y)}(\phi M, \psi N)=\mathrm{cl}_{(V, W)}(M, N) /(X, Y) .
$$

(g) If $(X, Y)$ is q.s. in the torsion-free system $(V, W)$, then $(X, Y)$ is torsionclosed in $(V, W)$.

Proof. Parts (a) and (b) are evident, while (c) is just a restatement of the definition of $t(V, W)$. Part (d) follows from (c) on noting that $(X, Y)$ is a torsionclosed subsystem of $(V, W)$ which includes $(M, N)$ if and only if $(X, Y) \supseteq[M, N]$ and $((V, W) /[M, N]) /((X, Y) /[M, N])$, which is isomorphic to $(V, W) /(X, Y)$, is torsionfree. Under the first hypothesis of (e), the system $(X, Y) /\left((X, Y) \cap \mathrm{cl}_{(V, W)}(M, N)\right)$, being isomorphic to the subsystem $\left((X, Y)+\operatorname{cl}_{(V, W)}(M, N)\right) / \mathrm{cl}_{(V, W)}(M, N)$ of $(V, W) / \mathrm{cl}_{(V, W)}(M, N)$ is torsion-free. Hence $(X, Y) \cap \mathrm{cl}_{(V, W)}(M, N)$ is a torsionclosed subsystem of $(X, Y)$ which includes $(M, N)$. Therefore it includes $\operatorname{cl}_{(X, Y)}(M, N)$. If in addition $(V, W) /(X, Y)$ is torsion-free, then since $(X, Y) / \mathrm{cl}_{(X, Y)}(M, N)$ is torsion-free it follows immediately that $(V, W) / \mathrm{cl}_{(X, Y)}(M, N)$ is torsion-free. Therefore since $\operatorname{cl}_{(X, Y)}(M, N)$ contains $(M, N)$, it contains $\mathrm{cl}_{(V, W)}(M, N)$. Part (f) follows from the fact that a subsystem which contains $(X, Y)$ is torsion-closed in $(V, W)$ and contains $(M, N)$ if and only if its homomorphic image is torsion-closed in $(V, W) /(X, Y)$ and contains $(\phi M, \psi N)$. Finally, under the assumptions of (g), if $C v \in Y$, where $v \in V, 0 \neq C \in \mathscr{T}$, and $(\pi, \rho)$ is a projection of $(X, Y)+([v], \mathscr{T}[v])$ onto $(X, Y)$, then $C v=\rho C v=C \pi v$. Hence $v=\pi v \in X$.

Let $(V, W)$ be a system, $w$ an element of $W$ and $L$ a subset of $W$. We say that $w$ depends on $L$ (in symbols: $w \prec L$ ) if and only if $w$ belongs to the range space of $\mathrm{cl}_{(V, W)}(\varnothing, L)$. This notion is of course relative to the system $(V, W)$. However, 2.1(e) shows that if $(X, Y)$ is a subsystem of $(V, W)$ and an element of $Y$ depends on a subset of $Y$ in $(X, Y)$, it does so in $(V, W)$ too. In Theorem 2.3 below we shall show that the relation we have defined satisfies the usual Steinitz-van der Waerden dependence postulates.

2.2. LEMmA. If $(V, W)$ is a torsion-free system, the following assertions are equivalent:

(a) $(V, W)$ is generated by a nonzero element $w$ of $W$.

(b) $(V, W) \neq(0,0)$, and every finite-dimensional subsystem of $(V, W)$ is contained in a subsystem of some type $\mathrm{III}_{m}$.

(c) $(V, W) \neq(0,0)$ and every homomorphic image of $(V, W)$ with a non-null kernel is a torsion system.

Proof. The implication (a) $\Rightarrow$ (b) appeared implicitly in the proof of one of the lemmas of [1]. For the sake of completeness we include a proof here. Assertion (a) means that $(V, W) /(0,[w])$ is a torsion system. Hence by 1.11 , if $(X, Y)$ is any finite-dimensional system, then $((X, Y)+(0,[w])) /(0,[w])$ is included in a finite-dimensional torsion subsystem $(U, Z) /(0,[w])$ of $(V, W) /(0,[w])$. According to 1.7 we have a decomposition 


$$
(U, Z) /(0,[w])=\Sigma+\left(U_{i}, Z_{i}\right) /(0,[w])
$$

where the subsystems $\left(U_{i}, Z_{i}\right) /(0,[w])$ are of the types $\mathrm{I}_{m_{i}}, \mathrm{II}_{m_{i}}^{\theta_{i}}$ or $\mathrm{III}_{m_{i}}$. A subsystem $\left(U_{i}, Z_{i}\right) /(0,[w])$ cannot be of type $\mathrm{III}_{m_{i}}$ since it is a homomorphic image of the torsion system $(U, Z) /(0,[w])$. Were it of type $I_{m_{i}}$, we would get $\operatorname{dim} U_{i}=m_{i}+1=\operatorname{dim} Z_{i} /[w]+1=\operatorname{dim} Z_{i}$; which, since the complex field is algebraically closed, implies the existence of an eigenvector in $\left(U_{i}, Z_{i}\right)$ against the assumption that $(V, W)$ is torsion-free. Hence all the summands are of the types $\mathrm{II}_{m_{i}}^{\theta_{i}}$ and therefore $\operatorname{dim} Z=\Sigma\left(m_{i}+1\right)+1=\operatorname{dim} U+1$. It follows that in a complete decomposition of the torsion-free system $(U, Z)$, all the summands of which are of the types III $_{m_{j}}$, only one summand appears. Thus $(X, Y)$ is contained in the subsystem $(U, Z)$ which is of type III $_{m}$. Suppose now that (b) holds and let $(X, Y) \neq(0,0)$ be a subsystem of $(V, W)$. Since $(V, W)$ is torsion-free, $Y$ contains a nonzero element $w$. Given any element of $V$ or $W$ there exists by our assumption a subsystem $(U, Z)$ of some type III $_{m}$ which contains $w$ and the given element. In a decomposition $\left({ }^{*}\right)$ no summand is of type $I_{m_{t}}$ by the above argument. Since $\operatorname{dim} U / 0=m+1=\operatorname{dim} Z /[w]$ and $\operatorname{dim} U_{i} / 0 \leqq \operatorname{dim} Z_{i} /[w]$, we have equalities throughout; i.e., the summands are of the types $\mathrm{II}_{\boldsymbol{m}_{i}}^{\boldsymbol{\theta}_{i}}$. It follow that $(V, W) /(0,[w])$ is a sum of subsystems of types $\mathrm{II}_{m}{ }_{m}^{\theta}$; hence a torsion system. Therefore

$$
(V, W) /(X, Y) \cong((V, W) /(0,[w])) /((X, Y) /(0,[w]))
$$

is also a torsion system. Finally if (c) holds and $w$ is any nonzero element of $W$, then evidently $(V, W)=\operatorname{cl}(\varnothing,\{w\})$.

2.3. TheORem. Let $(V, W)$ be a system, $L, M$ subsets of $W$ and $w$, $w_{i}$ elements of $W$. Then

(a) If $w \prec L$ and every element of $L$ depends on $M$, then $w \prec M$.

(b) $w \prec\{w\}$.

(c) If $w \prec L$ and $L \subseteq M$, then $w \prec M$.

(d) If $w \prec L$, then $w$ depends on a finite subset of $L$.

(e) If $w \prec\left\{w_{1}, \cdots, w_{n}\right\}$ and $w$ not $\prec\left\{w_{1}, \cdots, w_{n-1}\right\}$, then $w_{n} \prec\left\{w_{1}, \cdots, w_{n-1}, w\right\}$.

Proof. By the assumption of (a) we have $(\varnothing,\{l\}) \subseteq \operatorname{cl}(\varnothing, M)$ for every $l \in L$, hence $(\varnothing, L) \subseteq \operatorname{cl}(\varnothing, M)$. Therefore $(\varnothing,\{w\}) \subseteq \operatorname{cl}(\varnothing, L) \subseteq \operatorname{cl} \operatorname{cl}(\varnothing, M)=\operatorname{cl}(\varnothing, M)$ and $w \prec M$. Now, if $w$ depends linearly on $L$, then $w \prec L$ since the range space of $\operatorname{cl}(\varnothing, L)$ is a subspace which contains $L$. This implies (b) and also shows that (c) follows from (a). To prove (d), we first observe that if $(X, Y) /(0,[L])$ is a subsystem of $(V, W) /(0,[L])$ which is of type $\mathrm{II}_{m}^{\theta}$ or $\mathrm{I}_{0}$, then every element of $Y$ depends on a finite subset of $L$. To see this, say in the case $\mathrm{II}_{m}^{\boldsymbol{\theta}}, \theta \neq \infty$, consider a chain $\left\{x_{k}\right\} \in C_{0}^{0}{ }_{m}^{\infty}\left(A, B_{\theta} ; X / 0, Y / L\right)$ which spans $(X, Y) /(0,[L])(1.6)$. Then we have $A x_{k}=y_{k}, B_{\theta} x_{k}=y_{k+1}+l_{k+1}, k=0, \cdots, m$, with $y_{m+1}=0$ and $l_{k} \in[L]$. Any given element $y$ of $Y$ is of the form $y=\sum_{k=1}^{m} \alpha_{k} y_{k}+l$, where $l \in[L]$. There exists a finite subset $L^{\prime}$ of $L$ such that $l_{k}, l \in\left[L^{\prime}\right], k=1, \cdots, m+1$. 
Hence $\left\{x_{k}\right\} \in C_{0}^{0}{ }_{m}^{\infty}\left(A, B_{\theta} ; X / 0, Y /\left[L^{\prime}\right]\right)$ spans a subsystem of $(X, Y) /\left(0,\left[L^{\prime}\right]\right)$ or of $(V, W) /\left(0,\left[L^{\prime}\right]\right)$ which contains $y+\left[L^{\prime}\right]$. This subsystem is a homomorphic image of a system of type $\mathrm{II}_{m}^{\theta}$ and is therefore a torsion system. Thus, by 1.12, $\left(\varnothing,\left\{y+\left[L^{\prime}\right]\right\}\right) \subseteq t\left((V, W) /\left(0,\left[L^{\prime}\right]\right)\right.$ and $y \prec L^{\prime}$. Now if $w \prec L$, then $w+[L]$ belongs to the range space of the torsion system $\mathrm{cl}(0, L) /(0,[L])$. By $1.11, w+[L]$ is in the range space of a finite sum $\sum_{j=1}^{p}\left(X_{j}, Y_{j}\right) /(0,[L])$ of subsystems of the types $\mathrm{II}_{m}^{\theta}$ or $\mathrm{I}_{0}$; i.e., $w=\sum_{j=1}^{p} y_{j}+\sum_{k=1}^{q} \alpha_{k} l_{k}$ where $l_{k} \in L$ and $y_{j} \in Y_{j}$. By what we have observed, there exist finite subsets $L_{j}$ of $L$ such that $y_{j} \prec L_{j}, j=1, \cdots, p$. Since $w$ depends linearly on the set $\left\{y_{1}, \cdots, y_{p}, l_{1}, \cdots, l_{q}\right\}$ every element of which depends on $L^{\prime}=\left\{l_{1}, \cdots, l_{q}\right\} \cup \bigcup_{j=1}^{p} L_{j}$, we have $w \prec L^{\prime}$ where $L^{\prime}$ is a finite subset of $L$. For part (e) let $\left(X_{1}, Y_{1}\right)=\operatorname{cl}\left(\varnothing,\left\{w_{1}, \cdots, w_{n}\right\}\right),\left(X_{2}, Y_{2}\right)=\operatorname{cl}\left(\varnothing,\left\{w_{1}, \cdots, w_{n-1}\right\}\right)$, $\left(X_{3}, Y_{3}\right)=\operatorname{cl}\left(\varnothing,\left\{w_{1}, \cdots, w_{n-1}, w\right\}\right)$ and let $(\phi, \psi)$ denote the natural homomorphism of $(V, W)$ onto $(V, W) /\left(X_{2}, Y_{2}\right)$. We have that $\left(X_{2}, Y_{2}\right) \subseteq\left(X_{1}, Y_{1}\right)$, $\left(X_{1}, Y_{1}\right) /\left(X_{2}, Y_{2}\right)$ is torsion-closed in $(V, W) /\left(X_{2}, Y_{2}\right)$ and $\psi w_{i}=\psi 0$ for $i=1, \cdots$, $n-1$. Therefore by $2.1(\mathrm{e})(\mathrm{f})$ we obtain

$$
\begin{aligned}
\operatorname{cl}_{\left(X_{1}, Y_{1}\right) /\left(X_{2}, Y_{2}\right)}\left(\varnothing,\left\{\psi w_{n}\right\}\right) & =\operatorname{cl}_{(V, W) /\left(X_{2}, Y_{2}\right)}\left(\varnothing,\left\{\psi w_{n}\right\}\right) \\
& =\operatorname{cl}_{(V, W) /\left(X_{2}, Y_{2}\right)}\left(\varnothing,\left\{\psi w_{1}, \cdots, \psi w_{n-1}, \psi w_{n}\right\}\right) \\
& =\left(X_{1}, Y_{1}\right) /\left(X_{2}, Y_{2}\right) .
\end{aligned}
$$

Thus the system $\left(X_{1}, Y_{1}\right) /\left(X_{2}, Y_{2}\right)$ which is evidently torsion-free is generated by $\psi w_{n}$. From parts (a),(b),(c), and our hypotheses it follows that $w_{n}$ not $\prec$ $\left\{w_{1}, \cdots, w_{n-1}\right\}$; hence $\psi w_{n} \neq \psi 0$. Similarly $\psi w \neq \psi 0$ and therefore we conclude from the equivalence of (a) and (c) in Lemma 2.2 that $\psi w$ also generates $\left(X_{1}, Y_{1}\right) /\left(X_{2}, Y_{2}\right)$. But again by $2.1(\mathrm{e})(\mathrm{f})$

$$
\begin{aligned}
\operatorname{cl}_{\left(X_{1}, Y_{1}\right) /\left(X_{2}, Y_{2}\right)}(\varnothing,\{\psi w\}) & =\operatorname{cl}_{(V, W) /\left(X_{2}, Y_{2}\right)}(\varnothing,\{\psi w\}) \\
& =\operatorname{cl}_{(V, W) /\left(X_{2}, Y_{2}\right)}\left(\varnothing,\left\{\psi w_{1}, \cdots, \psi w_{n-1}, \psi w\right\}\right) \\
& =\left(X_{3}, Y_{3}\right) /\left(X_{2}, Y_{2}\right) .
\end{aligned}
$$

Hence $\left(X_{1}, Y_{1}\right)=\left(X_{3}, Y_{3}\right)$ and $w_{n} \in Y_{3}$ as required.

From Theorem 2.3 follow the well-known results (see e.g. [7]) that every independent subset (in particular the void set) of the range space of a system $(V, W)$ is contained in a basis with respect to generation (i.e., an independent subset which generates $(V, W))$, that any subset of $W$ which generates $(V, W)$ contains a basis and that any two such bases of $(V, W)$ have the same cardinality. This common cardinal will be called the rank of $(V, W)$ and denoted rank $(V, W)$. The rank is obviously an isomorphism invariant of systems. A more precise term would have been "torsion-free rank" because of the following theorem.

2.4. THEOREM. We have $\operatorname{rank}(V, W) \leqq \operatorname{rank}(V, W) /(X, Y)+\operatorname{rank}(X, Y)$ for every subsystem $(X, Y)$ of $(V, W)$ with equality if $(X, Y)$ is torsion-closed in $(V, W)$. In particular rank $(V, W)=\operatorname{rank}(V, W) / t(V, W)$. 
The proof is easily obtained using Lemma 2.1 and Theorem 2.3. We omit the details. Lemma 2.2 can now be viewed as a characterization of torsion-free systems of rank 1. By similar arguments one may show that if a torsion-free system $(V, W)$ is of finite rank $r$, then every finite-dimensional system is contained in a direct sum of $r$ subsystems of types III $_{m}$. Conversely, if the latter condition holds, then $\operatorname{rank}(V, W) \leqq r$.

3. Torsion-free systems of rank 1. We are interested in these systems mainly because they are the simplest q.s.irr. torsion-free systems. This is a corollary of the characterization given in Lemma 2.2(c). In fact, if $(X, Y)$ is a non-null q.s. subsystem of a torsion-free system of rank $1(V, W)$, then by $2.2(\mathrm{c}),(V, W) /(X, Y)$ is a torsion system. By $2.1(\mathrm{~g})$ it is also torsion-free. Hence, $1.11,(V, W) /(X, Y)$ is a null system; i.e., $(X, Y)=(V, W)$. Another corollary is the following

3.1. Lemma. A homomorphism $(\phi, \psi)$ of a torsion-free system of rank 1 $(V, W)$ into a torsion-free system $(X, Y)$ either vanishes or is a monomorphism.

Proof. We have the factorization $(\phi, \psi)=(\tilde{\phi}, \tilde{\psi})(\mu, v)$ where $(\mu, v)$ is the natural homomorphism of $(V, W)$ onto $(V, W) / \operatorname{Ker}(\phi, \psi)$ and $(\tilde{\phi}, \tilde{\psi})$ is the homomorphism of $(V, W) / \operatorname{Ker}(\phi, \psi)$ into $(X, Y)$ induced by $(\phi, \psi)$. If $\operatorname{Ker}(\phi, \psi) \neq(0,0)$, then by $2.2(\mathrm{c})(V, W) / \operatorname{Ker}(\phi, \psi)$ is a torsion system. Therefore, by 1.10 , $(\phi, \psi)(V, W)=(\tilde{\phi}, \tilde{\psi})((V, W) / \operatorname{Ker}(\phi, \psi))$ is a torsion system. Being a subsystem of $(X, Y),(\phi, \psi)(V, W)$ is also torsion-free. Hence $(\phi, \psi)(V, W)=(0,0)$; i.e., $(\phi, \psi)=(0,0)$.

Guided by this result and using 2.2(b) and the chain representation of systems of type III $_{m}(1.6)$, one proves the following theorem. Since it is one of the lemmas in [1] with only terminology changes, we omit the details.

3.2. THEOREM. Every torsion-free system of rank $1(V, W)$ is isomorphic to a subsystem of a system of type $\mathscr{R}$. In more detail, given an element $w \neq 0$ of $W$, a nonzero rational function $f$ and a basis $A, B$ of $\mathscr{T}$, there exists one and only one monomorphism $(\phi, \psi)$ of $(V, W)$ into $(R, R)_{A, B}$ such that $\psi w=f$.

Our next aim is to give a detailed description of torsion-free systems of rank 1 and then use it to characterize their isomorphism types. We prefer to do this in a terminology which does not presuppose embedding in a system of type $\mathscr{R}$. We decompose the set of all ordered bases of $\mathscr{T}$ into classes of proportional pairs, and obtain a set $\mathscr{M}$ of classes which is in a natural 1-1 correspondence with the set of all Moebius transformations. Let $A / B$ denote the class of the basis $A, B$. By convention $A / B_{\infty}$ will stand for $B / A$. We denote by $\mathscr{P}[\mathscr{M}]$ the set of all formal polynomials

$$
\alpha_{0}+\sum_{j=1}^{l} \alpha_{j}\left(A_{j} / B_{j}\right)^{k_{j}}
$$


where $A_{j} / B_{j} \in \mathscr{M}, \alpha_{j}$ are complex numbers and $k_{j}$ are non-negative integers. These constitute a complex vector space with the natural definitions of equality and operations (we stipulate that $\alpha\left(A / B^{0}\right)=\alpha$ for all $A / B \in \mathscr{M}, \alpha$ complex). Let $(V, W)$ be a system and let $w \in W$. We say that the monomial $(A / B)^{k}$ is applicable to $w$ (in $(V, W)$ ) if and only if $k=0$ or there exists a chain $\left\{v_{i}\right\}_{-k}^{-1}$ in $C_{-k-1}^{-k}(A, B ; V, W)$ such that $B v_{-1}=w$. This property is obviously independent of the base chosen from the class $A / B$. A polynomial in $\mathscr{P}[\mathscr{M}]$ is said to be applicable to $w$ if it vanishes or, when written in terms of distinct monomials with nonvanishing coefficients, all its monomials are applicable to $w$. If $(V, W)$ is torsion-free and the polynomial $(*)$, with $\alpha_{j} \neq 0$ for $1 \leqq j \leqq l$, is applicable to $w$, then the range elements of the chains $\left\{v_{i}^{j}\right\}_{-k_{j}}^{-1}$ in $C_{-k_{j} j-1}^{-k_{j}}\left(A_{j}, B_{j} ; V, W\right)$ such that $B_{j} v_{-1}^{j}=w$ are uniquely determined, and we define

$$
\left(\alpha_{0}+\sum \alpha_{j}\left(A_{j} / B_{j}\right)^{k_{j}}\right) w=\alpha_{0} w+\sum \alpha_{j} w_{-k_{j}}^{j} .
$$

The value assigned by $\left({ }^{* *}\right)$ is then independent of the mode of writing the polynomial $(*)$ or of the bases chosen from the classes $A_{j} / B_{j}$.

3.3. THEOREM. A non-null torsion-free system $(V, W)$ is of rank 1 if and only if there exists an element $w$ in $W$ such that for every $z$ in $W$ there is a polynomial $\alpha_{0}+\sum_{j=1}^{l} \alpha_{j}\left(A_{j} / B_{j}\right)^{k_{j}}$ in $\mathscr{P}[\mathscr{M}]$ with

$$
\left(\alpha_{0}+\sum_{j=1}^{l} \alpha_{j}\left(A_{j} / B_{j}\right)^{k_{j}}\right) w=z .
$$

If $(V, W)$ is torsion-free of rank 1 , then every $0 \neq w \in W$ has this property.

Proof. Suppose that $(V, W)$ is torsion-free of rank $1, w, z \in W$ and $w \neq 0$. By 2.2 (b) there exists a subsystem $(U, Z)$ of some type $\mathrm{III}_{m}$ such that $w, z \in Z$. As in the proof that $2.2(\mathrm{~b})$ implies $2.2(\mathrm{c})$, we have $(U, Z) /(0,[w])=\Sigma \dot{+}\left(X^{j}, Y^{j}\right)$, where $\left(X^{j}, Y^{j}\right)$ are all of the respective types $\mathrm{II}_{m_{j}}^{\theta_{j}}$. Let $\left\{z_{k}^{j}\right\}_{k=-m_{j}}^{\infty}$ be sequences of elements of $Z$ such that the sequences $\left\{z_{k}^{j}+[w]\right\}_{-m_{j}}^{\infty}$ are the range elements of chains in $C_{-m_{j}}^{-m_{j}}{ }_{0}^{\infty}\left(A, B_{\theta_{j}} ; X_{j}, Y_{j}\right)$ which span $\left(X^{j}, Y^{j}\right)$. (Cf. 1.6. It is convenient to shift the indices here.) Then $z$ is of the form $z=\beta_{0} w+\sum_{j} \sum_{k=-m_{j}}^{0} \beta_{k}^{j} z_{k}^{j}$. Since the set of polynomials applicable to $w$ is a subspace of $\mathscr{P}[\mathscr{M}]$ and since the mapping $w \rightarrow p w, p$ in this subspace, is linear in $p$, it suffices to show that each $z_{k}^{j}$ is of the form given in the theorem. To this end we check that if $\left\{z_{k}\right\}_{-m}^{\infty}$ is a sequence in $Z$ such that $\left\{z_{k}+[w]\right\}$ are the range elements of a proper chain $\left\{u_{k}\right\}$ in $C_{-m}^{-m} \underset{0}{\infty}\left(A, B_{\theta} ; U / 0, Z /[w]\right)$ (we choose, as we may, $z_{k}=w$ for $k \geqq 1$ ), then $z_{-m}, m \geqq-1$, is of the form

$$
z_{-m}=\left(\left(A / B_{\theta}\right)^{m+1}+\alpha_{1 m}\left(A / B_{\theta}\right)^{m}+\cdots+\alpha_{m m} A / B_{\theta}+\alpha_{m+1, m}\right) w .
$$

We assume $\theta \neq \infty$; otherwise change the roles of $A$ and $B$. The assertion being obviously true for $m=-1$, consider the case $m+1$ under the inductive assump- 
tion that is true in the case $m(m \geqq-1)$. Then $z_{-m}$ is of the form (3.3.1). From the chain relations there exist $\alpha, \beta$ such that

$$
\begin{aligned}
z_{-m}+\alpha w & =B_{\theta} u_{-m-1}, \\
z_{-m-1} & =A u_{-m-1}+\beta w .
\end{aligned}
$$

By (3.3.2), $A / B_{\theta}$ is applicable to $z_{-m}+\alpha w$, and

$$
A u_{-m-1}=\left(A / B_{\theta}\right)\left(z_{-m}+\alpha w\right) .
$$

Therefore by (3.3.3)

$$
z_{-m-1}=\left(A / B_{\theta}\right)\left(z_{-m}+\alpha w\right)+\beta w .
$$

We have $B_{\theta} u_{0}=\gamma w$ with $\gamma \neq 0$ since $u_{0} \neq 0$ and $(V, W)$ is torsion-free. Therefore $A / B_{\theta}$ is applicable to $w$, and hence by (3.3.4) also to $z_{-m}$. Since, by (3.3.1), $\left(A / B_{\theta}\right)^{m+1}$ is applicable tow, it follows that $A / B_{\theta}$ is applicable to $z_{-m}-\left(A / B_{\theta}\right)^{m+1} w$. Hence $\left(A / B_{\theta}\right)^{m+2}$ is applicable to $w$. Therefore (3.3.5) may be written

$$
z_{-m-1}=\left(\left(A / B_{\theta}\right)^{m+2}+\alpha_{1 m}\left(A / B_{\theta}\right)^{m+1}+\cdots+\left(\alpha_{m+1, m}+\alpha\right) A / B_{\theta}+\beta\right) w,
$$

which proves our statement.

Assume now that an element $w$ with the property of the theorem exists. Every element of the form $(A / B)^{k} w+[w], A / B \in \mathscr{M}$, is contained in the range space of a torsion subsystem of $(V, W) /(0,[w])$. Therefore it follows from our assumption that the range space of $t((V, W) /(0,[w]))$ is the whole of $W /[w]$. Hence, if $v \in V$ and $A, B$ is a basis of $\mathscr{T}$, then $A v+[w], B v+[w]$ belong to the range space of a finite-dimensional torsion subsystem $(U, Z) /(0,[w])$. As in the proof that 2.2(a) implies $2.2($ b), we have $\operatorname{dim} U+1=\operatorname{dim} Z$. Hence, if $v$ did not belong to $U$, the subsystem $(U+[v], Z)$ would have domain and range space of the same dimension, which contradicts the fact that $(V, W)$ is torsion-free. It follows that

$$
t((V, W) /(0,[w]))=(V, W) /(0,[w]) \text { or } \quad(V, W)=\operatorname{cl}(\varnothing,[w]) .
$$

We now define complete isomorphism invariants for torsion-free systems of rank 1 . Let $(V, W)$ be any system, $w \in W$. It is easy to verify that if $A, B$ and $A^{\prime}, B^{\prime}$ are bases of $\mathscr{T}$ such that $B^{\prime}$ is a multiple of $B$, then $(A / B)^{k}$ is applicable to $w$ if and only if $\left(A^{\prime} / B^{\prime}\right)^{k}$ is. This justifies the following definition. If $k$ is the largest non-negative integer for which $(A / B)^{k}$ is applicable to $w$, we call $k$ the height of $w$ at the class $\dot{B}$ of multiples of $B$, and denote it by $H(w)_{\dot{B}}$; if no such maximal integer exists, we define $H(w)_{\dot{B}}=\infty$. Clearly the height is defined relative to the system $(V, W)$. If $w \in Y$ where $(X, Y)$ is a subsystem of $(V, W)$, then $H(W)_{\dot{B}}$ relative to $(X, Y)$ does not exceed $H(w)_{\dot{B}}$ relative to $(V, W)$, with equality $\operatorname{if}(X, Y)$ is torsion-closed in $(V, W)$. If a basis $A, B$ is fixed during a discussion, we speak of the height at $\theta$ instead of the height at $B_{0}$ and denote $H(w)_{B_{\theta}}=H(w)_{\mu}$. A triplet 
$(A, B, H)$ where $A, B$ is a basis of $\mathscr{T}$ and $H$ is a function from $\Theta$ into the union of the non-negative integers and the symbol $\infty$ will be called a height function ( $A, B$ omitted when understood). Thus the function $H(w): \theta \rightarrow H(w)_{\theta}$ is "the height function of $w$ '. We introduce an equivalence relation $\sim$ in the set of height functions (with a fixed basis) by defining $H \sim H^{\prime}$ if and only if :

(a) The set $\Delta=\left\{\theta \in \Theta \mid H_{\theta} \neq H_{\theta}^{\prime}\right\}$ is finite and $H_{\theta} \neq \infty \neq H_{\theta}^{\prime}$ for $\theta \in \Delta$;

(b) If one of the functions does not assume the value $\infty$ (then both do not by (a)), then $\sum_{\theta \in \Delta} H_{\theta}=\sum_{\theta \in \Delta} H_{\theta}^{\prime}$.

A relation of equality, inequality or equivalence between the height functions of two elements is clearly invariant under a change of the basis $A, B$. The class of height functions equivalent to $H(w)$ will be called the type of $w$ and denoted by $\mathscr{H}(w)$. The next theorem justifies defining the type $\mathscr{H}(V, W)$ of a torsion-free system of rank $1(V, W)$ to be $\mathscr{H}(w)$ where $w$ is any nonzero element of $W$.

3.4. THEOREM. If $(V, W)$ is a torsion-free system of rank 1 , then the height functions of nonzero elements of $W$ form a complete equivalence class. The mapping $(V, W) \rightarrow \mathscr{H}(V, W)$ induces a 1-1 correspondence between the set of all isomorphism types of torsion-free systems of rank 1 and the set of all types.

Proof. Since the height function of an element is obviously an isomorphism invariant, to prove the first part we may assume according to Theorem 3.2 that $(V, W)$ is a subsystem of $(R, R)_{A, B}$ such that the function $w=1$ belongs to $W$. From Theorem 3.3, the remark justifying the definition of height and the uniqueness of the representation of a rational function by partial fractions it follows that

$$
W=\left[\left\{(\zeta-\theta)^{-k} \mid k \text { integer, } 0 \leqq k<H(w)_{\theta}+1, \theta \in \Theta\right\}\right],
$$

where $(\zeta-\theta)^{-k}$ denotes $\zeta^{k}$ when $\theta=\infty$, and

$$
V=\left[\left\{(\zeta-\theta)^{-k} \mid 0<k<H(w)_{\theta}+1, \theta \neq \infty\right\}\right]+\left[\left\{\zeta^{k} \mid 0 \leqq k<H_{\infty}(w)\right\}\right] .
$$

Thus if $\mathscr{W}(f)_{\theta}$ denotes the order of the rational function $f$ at $\theta$ (usual convention at $\theta=\infty$ ), then

$$
W=\left\{w^{\prime} \in R \mid-\mathscr{W}\left(w^{\prime}\right)_{\eta} \leqq H(w)_{\eta} \text { for every } \eta \in \Theta\right\} .
$$

For $0 \neq w^{\prime} \in W$ we have

$$
H\left(w^{\prime}\right)_{\theta}=\sup \left\{k \mid k \text { integer, }-\mathscr{W}\left((\zeta-\theta)^{-k} w^{\prime}\right)_{\eta} \leqq H(w)_{\eta} \text { for every } \eta \in \Theta\right\} .
$$

By (3.4.3), we need to consider only non-negative integers $k$ in (3.4.4). Since $\mathscr{W}(f g)=\mathscr{W}(f)+\mathscr{W}(g)$,

$$
-\mathscr{W}\left((\zeta-\theta)^{-k}\right)_{\eta}=\left\{\begin{array}{lc}
k & \text { if } \eta=\theta \\
0 \text { or }-k & \text { otherwise }
\end{array}\right.
$$

and $\mathscr{W}\left(w^{\prime}\right)_{\eta}$ is finite-valued, it follows that a non-negative integer $k$ satisfies the condition of (3.4.4) if and only if $k \leqq H(w)_{\theta}+\mathscr{W}\left(w^{\prime}\right)_{\theta}$; i.e., 


$$
H\left(w^{\prime}\right)_{\theta}=H(w)_{\theta}+\mathscr{W}\left(w^{\prime}\right)_{\theta} .
$$

Hence $H\left(w^{\prime}\right)$ and $H(w)$ differ only on the subset of the finite set $\Gamma=\left\{\theta \mid \mathscr{W}\left(w^{\prime}\right)_{\theta} \neq 0\right\}$ where both are finite; and since $\sum_{\theta \in \Gamma} \mathscr{W}\left(w^{\prime}\right)_{\theta}=0$, we have $H\left(w^{\prime}\right) \sim H(w)$.

Suppose conversely that $H^{\prime}$ is a height function equivalent to $H(w)$. Then the function $\mathscr{W}$ defined by $\mathscr{W}_{\theta}=H_{\theta}^{\prime}-H(w)_{\theta}$ (here $\infty-\infty=0$ ) is integer-valued and vanishes outside the finite set $\Delta=\left\{\theta \mid H_{\theta}^{\prime} \neq H(w)_{\theta}\right\}$. If $H(w)$ does not assume the value $\infty$, we have $\Sigma_{\theta \in \Delta} \mathscr{W}_{\theta}=0$. Hence there exists a rational function $w^{\prime}$ such that $\mathscr{W}\left(w^{\prime}\right)_{\theta}=\mathscr{W}_{\theta}, \theta \in \Theta$. Since $H_{\theta}^{\prime} \geqq 0$, we have $-\mathscr{W}\left(w^{\prime}\right)_{\theta} \leqq H(w)_{\theta}$ for every $\theta$. Thus, by (3.4.3), $w^{\prime} \in W$, and by (3.4.5) $H\left(w^{\prime}\right)=H^{\prime}$. If $H(w)$ does assume the value $\infty$, say at $\eta$, modify the definition of $\mathscr{W}$ at $\eta$ by $\mathscr{W}_{\eta}=-\Sigma_{\theta \in \Delta} \mathscr{W}_{\theta}$ and proceed as in the previous case. This proves our first statement.

By what we have shown, $\mathscr{H}(V, W)$ is well defined and depends only on the isomorphism type of $(V, W)$. If $\mathscr{H}(V, W)=\mathscr{H}\left(V^{\prime}, W^{\prime}\right)$, let $w, w^{\prime}$ be nonzero elements of $W, W^{\prime}$ respectively. Then $H(w) \sim H\left(w^{\prime}\right)$, and there exists an element $w^{\prime \prime}$ in $W$ such that $H\left(w^{\prime \prime}\right)=H\left(w^{\prime}\right)$. By $3.2,(V, W)$ and $\left(V^{\prime}, W^{\prime}\right)$ can be embedded in $(R, R)_{A, B}$ so that $w^{\prime \prime}$ and $w$ go as range elements into 1. Hence by (3.4.1), (3.4.2) $(V, W)$ and $\left(V^{\prime}, W^{\prime}\right)$ are mapped onto the same subsystem of $(R, R)_{A, B}$ and are therefore isomorphic. Finally, given a height function $H$, consider the subsystem $(V, W)$ of $(R, R)_{A, B}$ defined as in (3.4.1), (3.4.2) in terms of $H$ instead of $H(w)$. For the purpose of later reference we denote this system $(V, W)$ also by $(S, T)_{A, B}^{\boldsymbol{H}}=\left(S_{A, B}^{\boldsymbol{H}}, T_{A, B}^{\boldsymbol{H}}\right)$. Then $1 \in T_{A, B}^{\boldsymbol{H}}, H(1)=H$ and $w=1$ has the property of Theorem 3.3. Thus $(S, T)_{A, B}^{H}$ is torsion-free of rank 1 , and the mapping of the present theorem is onto the set of all types.

3.5. Corollary. A torsion-free system of rank $1(V, W)$ is nonsingular if and only if the height functions in $\mathscr{H}(V, W)$ assume the value $\infty$.

Proof. Since $(V, W)$ is torsion-free the mappings $v \rightarrow B_{\theta} v$ are always monomorphic. It is clear that $B_{\theta} V=W$ implies that $H(w)_{\theta}=\infty$ for every $w$ in $W$. Conversely, if $H \in \mathscr{H}(V, W)$ and $H_{\theta}=\infty$, then by $3.4, H(w)_{\theta}=\infty>0$ for every $w$ in $W$, so that every $w$ has a preimage under $B_{\theta}$.

We can introduce a natural partial order $\leqq$ among the types of torsion-free systems of rank 1: $\mathscr{H}^{1} \leqq \mathscr{H}^{2}$ if and only if a system of type $\mathscr{H}^{1}$ can be isomorphically embedded in a system of type $\mathscr{H}^{2}$. It is clear that this relation is reflexive and transitive. However, the fact that $\mathscr{H}^{1} \leqq \mathscr{H}^{2}$ and $\mathscr{H}^{2} \leqq \mathscr{H}^{1}$ implies $\mathscr{H}^{1}=\mathscr{H}^{2}$ requires verification since torsion-free systems of rank 1 may well have proper isomorphic subsystems (see 3.7). From the proof of 3.4 one sees that $\mathscr{H}^{1} \leqq \mathscr{H}^{2}$ holds if and only if there exist height functions $H^{i} \in \mathscr{H}^{i}$, $i=1,2$, such that $H^{1} \leqq H^{2}$. We show that if $H^{i}, \bar{H}^{i} \in \mathscr{H}^{i}, i=1,2, H^{1} \leqq H^{2}$, and $\bar{H}^{1} \geqq \bar{H}^{2}$, then $H^{1} \sim H^{2}$. Let $\Delta^{i}=\left\{\theta \in \Theta \mid H_{\theta}^{i} \neq \bar{H}_{\theta}^{i}\right\}, i=1,2$. Then obviously $\Delta=\left\{\theta \in \Theta \mid H_{\theta}^{1} \neq H_{\theta}^{2}\right\} \subseteq \Delta^{1} \cup \Delta^{2}$. Thus $\Delta$ is finite and the functions $H^{1}, H^{2}$ assume only finite values on $\Delta$. If $H^{1}$ does not assume the value $\infty$, the 
same holds for the equivalent function $\bar{H}^{1}$; hence also for $\bar{H}^{2}$ and its equivalent $H^{2}$. We then have the inequalities between finite numbers

$$
\sum_{\Delta^{1} \cup \Delta^{2}} H_{\theta}^{1} \leqq \sum_{\Delta^{1} \cup \Delta^{2}} H_{\theta}^{2}=\sum_{\Delta^{1} \cup \Delta^{2}} \bar{H}_{\theta}^{2} \leqq \sum_{\Delta^{1} \cup \Delta^{2}} \bar{H}_{\theta}^{1}=\sum_{\Delta^{1} \cup \Delta^{2}} H_{\theta}^{1} .
$$

Since $H^{1}$ and $H^{2}$ coincide on $\left(\Delta^{1} \cup \Delta^{2}\right)-\Delta$, it follows that $\Sigma_{\Delta} H_{\theta}^{1}=\Sigma_{\Delta} H_{\theta}^{2}$.

We now determine the endomorphisms of torsion-free systems of rank 1 .

3.6. THEOREM. Let $(V, W)$ be a torsion-free system of rank 1 and let $H \in \mathscr{H}(V, W)$. Then the algebra of endomorphisms of $(V, W)$ is isomorphic to the algebra of the rational functions $f$ such that $H_{\theta} \neq \infty$ implies $\mathscr{W}(f)_{\theta} \geqq 0$. More precisely, if $(V, W)$ is a subsystem of $(R, R)_{A, B}$ its endomorphisms are the multiplications $(f, f)$ (i.e., the pairs of mappings $v \rightarrow f v, w \rightarrow f w$ ) for $f$ as above.

Proof. The second statement implies the first one. Suppose therefore that $(V, W) \subseteq(R, R)_{A, B}$ and let $0 \neq w \in W$. If $(\phi, \psi)$ is an endomorphism of $(V, W)$, then the multiplication by $((\psi w) / w,(\psi w) / w)$ is a homomorphism of $(V, W)$ into $(R, R)_{A, B}$ which coincides on $w$ with $(\phi, \psi)$ considered as a homomorphism of $(V, W)$ into $(R, R)_{A, B}$. From 3.1 it follows that these mappings coincide on $(V, W)$; hence $(\phi, \psi)$ is a multiplication $(f, f)$. Now

$$
W=\left\{w^{\prime} \in R \mid-\mathscr{W}\left(w^{\prime}\right)_{\theta} \leqq H(w)_{\theta}-\mathscr{W}(w)_{\theta} \text { for every } \theta \in \Theta\right\} .
$$

(This reduces to (3.4.3) by the multiplication isomorphism $(1 / w, 1 / w)$.) Therefore for every non-negative integer $k$ we have

$$
\mathscr{W}\left(\psi^{k} w\right)_{\theta}=\mathscr{W}\left(f^{k} w\right)_{\theta}=k \mathscr{W}(f)_{\theta}+\mathscr{W}(w)_{\theta} \geqq-H(w)_{\theta}+\mathscr{W}(w)_{\theta} .
$$

Hence if $H_{\theta}<\infty$ then $H(w)_{\theta}<\infty$ and it follows that $\mathscr{W}(f)_{\theta} \geqq 0$. Conversely, if $f$ satisfies the stated condition and $0 \neq w^{\prime} \in W$, then $-\mathscr{W}\left(f w^{\prime}\right)_{\theta}=-\mathscr{W}(f)_{\theta}-\mathscr{W}\left(w^{\prime}\right)_{\theta}$ $\leqq H\left(w^{\prime}\right)_{\theta}-\mathscr{W}\left(w^{\prime}\right)_{\theta}$ for $H\left(w^{\prime}\right)_{\theta} \neq \infty$. Since this obviously holds if $H\left(w^{\prime}\right)_{\theta}=\infty$, we have $f w^{\prime} \in W$. Since, by (3.4.1) and (3.4.2), $V=\{v \in R \mid v, \zeta v \in W\}$, we have also $f V \subseteq V$. Thus $(f, f)$ is an endomorphism of $(V, W)$.

In view of the conditions given in $\$ 5$ for q.s. direct sums of torsion-free systems of rank 1, the following corollary of 3.6 will be of interest. We omit its straightforward verification.

3.7. COROLlARY. In the notation of 3.6, the automorphisms of $(V, W)$ are given by the multiplications $(f, f)$ where $H_{\theta} \neq \infty$ implies $\mathscr{W}(f)_{\theta}=0$. These together with $(0,0)$ form a field if and only if $(V, W)$ is of type $\mathscr{R}$ (field of all the rational functions) or there exists at most one $\theta$ in $\Theta$ with $H_{\theta}=\infty$ (field of scalar multiples of the identity). This field comprises all of $\operatorname{End}(V, W)$ if and only if $(V, W)$ is of type $\mathscr{R}$ or is singular.

4. Quasi-spectral direct sums. To discuss q.s. direct sums of subsystems of 
given isomorphism types in a system $(V, W)$, we choose for each isomorphism type a representative system $(S, T)$. Instead of subsystems of $(V, W)$ isomorphic to $(S, T)$, we consider monomorphisms of $(S, T)$ into $(V, W)$. A homomorphism $(\phi, \psi)$ of $(S, T)$ into $(V, W)$ is called a q.s. homomorphism in case $(\phi, \psi)(S, T)$ is q.s. in $(V, W)$. We denote by $D(S, T ; V, W)$ the subset of $\operatorname{Hom}(S, T ; V, W)$ consisting of those homomorphisms which are not q. s. monomorphisms. The vector space $\operatorname{Hom}(S, T ; V, W)$ is made into a right module over the algebra $\operatorname{End}(S, T)$ by setting $(\phi, \psi)(\mu, v)=(\phi \mu, \psi v), \quad(\phi, \psi) \in \operatorname{Hom}(S, T ; V, W)$, $(\mu, v) \in \operatorname{End}(S, T)$, where $(\phi \mu) s=\phi(\mu s),(\psi v) t=\psi(v t), s \in S, t \in T$. A subset $\left\{\left(\phi_{i}, \psi_{i}\right) \mid i \in I\right\}$ of $\operatorname{Hom}(S, T ; V, W)$ is said to be linearly dependent modulo $D(S, T ; V, W)$ in case there exist a nonvoid finite subset $\left\{\left(\phi_{i}, \psi_{i}\right) \mid i \in J\right\}, J \subseteq I$, and endomorphisms $\left\{\left(\mu_{i}, v_{i}\right) \mid i \in J\right\}$ of $(S, T)$ at least one of which is an automorphism, such that $\sum_{i \in J}\left(\phi_{i}, \psi_{i}\right)\left(\mu_{i}, v_{i}\right) \in D(S, T ; V, W)$. As we shall see below, this property does not always satisfy all that is usually demanded of linear dependence. We show first its connection with q.s. direct sums.

4.1. Lemma. Let $(S, T)$ and $(V, W)$ be arbitrary systems. Let $\left\{\left(\phi_{i}, \psi_{i}\right) \mid i \in I\right\}$ be a subset of $\operatorname{Hom}(S, T ; V, W)$ consisting of monomorphisms such that the subsystems $\left\{\left(\phi_{i}, \psi_{i}\right)(S, T) \mid i \in I\right\}$ are independent and the direct sum $\sum_{i \in I}+\left(\phi_{i}, \psi_{i}\right)(S, T)$ is q.s. in $(V, W)$. Then $\left\{\left(\phi_{i}, \psi_{i}\right) \mid i \in I\right\}$ is linearly independent modulo $D(S, T ; V, W)$.

Proof. Let $\left\{\left(\phi^{j}, \psi^{j}\right) \mid j=1, \cdots, n\right\}$ be a finite subset of $\left\{\left(\phi_{i}, \psi_{i}\right) \mid i \in I\right\}$, and consider a linear combination

$$
(\phi, \psi)=\sum_{j=1}^{n}\left(\phi^{j}, \psi^{j}\right)\left(\mu_{j}, v_{j}\right)
$$

where the $\left(\mu_{j}, v_{j}\right)$ are endomorphisms of $(S, T)$ at least one of which, say $\left(\mu_{1}, v_{1}\right)$, is an automorphism. We have to show that $(\phi, \psi)$ is a q.s. monomorphism. From (*) and

$$
\left(\phi^{1}, \psi^{1}\right)=(\phi, \psi)\left(\mu_{1}, v_{1}\right)^{-1}-\sum_{j=2}^{n}\left(\phi^{j}, \psi^{j}\right)\left(\mu_{j}, v_{j}\right)\left(\mu_{1}, v_{1}\right)^{-1}
$$

we obtain $(\phi, \psi)(S, T) \subseteq \sum_{j=1}^{n}\left(\phi^{j}, \psi^{j}\right)(S, T)$ and $\left(\phi^{1}, \psi^{1}\right)(S, T) \subseteq(\phi, \psi)(S, T)$ $+\sum_{j=2}^{n}\left(\phi^{j}, \psi^{j}\right)(S, T)$. Hence

$$
\sum_{j=1}^{n}\left(\phi^{j}, \psi^{j}\right)(S, T)=(\phi, \psi)(S, T)+\sum_{j=2}^{n}\left(\phi^{j}, \psi^{j}\right)(S, T) .
$$

If $\phi s_{1}+\sum_{j=2}^{n} \phi^{j} s_{j}=0, s_{j} \in S$, then $\phi^{1} \mu_{1} s_{1}+\sum_{j=2}^{n} \phi^{j}\left(\mu_{j} s_{1}+s_{j}\right)=0$. From the independence of the subspaces $\phi^{j} S, j=1, \cdots, n$, it follows that $\phi^{1} \mu_{1} s_{1}=0$. Since $\phi^{1}$ and $\mu_{1}$ are monomorphisms, we get $s_{1}=0$. This and $\left({ }^{* *}\right)$ show that $\phi S$ is a direct summand of $\sum_{j=1}^{n} \phi^{j} S$. The particular case $s_{j}=0, j=2, \cdots, n$, 
implies that $\phi$ is a monomorphism. The same argument applies to the range spaces, so that $(\phi, \psi)(S, T)$ is spectral in $\sum_{j=1}^{n}\left(\phi^{j}, \psi^{j}\right)(S, T)$ and $(\phi, \psi)$ is a monomorphism. Since $\sum_{j=1}^{n}\left(\phi^{j}, \psi^{j}\right)(S, T)$ is spectral in $\sum_{i \in I}\left(\phi_{i}, \psi_{i}\right)(S, T)$, which is q.s. in $(V, W)$, it follows from 1.1 and the fact that being spectral implies being q.s. that $(\phi, \psi)(S, T)$ is q.s. in $(V, W)$.

The next theorem reduces the question of the converse to the case of two homomorphisms. A similar argument appeared in [1] in a special case.

4.2. THEOREM. Let $\left\{\left(S_{j}, T_{j}\right)\right\}$ be a family of systems of distinct isomorphism types which satisfies the following condition:

If $(V, W)$ is an arbitrary system and $\left(\phi_{j}, \psi_{j}\right) \in \operatorname{Hom}\left(S_{j}, T_{j} ; V, W\right),\left(\phi_{h}, \psi_{h}\right)$ $\in \operatorname{Hom}\left(S_{h}, T_{h} ; V, W\right)$ are q.s. monomorphisms, which in case $j=h$ are also linearly independent modulo $D\left(S_{j}, T_{j} ; V, W\right)$, then $\left(\phi_{j}, \psi_{j}\right)\left(S_{j}, T_{j}\right)+\left(\phi_{h}, \psi_{h}\right)\left(S_{h}, T_{h}\right)$ is a direct sum which is q.s. in $(V, W)$.

Suppose that $(V, W)$ is a system and that for each $j,\left\{\left(\phi_{i j}, \psi_{i j}\right) \mid i \in I(j)\right\}$ is a subset of $\operatorname{Hom}\left(S_{j}, T_{j} ; V, W\right)$ (the index set $I(j)$ may be void). Then in order that the $\left(\phi_{i j}, \psi_{i j}\right)$ be monomorphisms, the systems $\left(\phi_{i j}, \psi_{i j}\right)\left(S_{j}, T_{j}\right)$ be independent and the direct sum $\Sigma_{j} \sum_{i \in I(j)}+\left(\phi_{i j}, \psi_{i j}\right)\left(S_{j}, T_{j}\right)$ be q.s. in $(V, W)$ it is necessary and sufficient that for each $j$ the set $\left\{\left(\phi_{i j}, \psi_{i j}\right) \mid i \in I(j)\right\}$ be linearly independent modulo $D\left(S_{j}, T_{j} ; V, W\right)$.

Proof. The necessity follows from the last lemma. If the linear independence condition holds, the fact that the $\left(\phi_{i j}, \psi_{i j}\right)$ are monomorphisms is obvious. The rest of the sufficiency proof is reduced by 1.2 to the case of a finite number $n$ of homomorphisms. If $n$ is 0,1 or 2 there is nothing to prove. Suppose that $n>2$ and that the statement is true in general for less than $n$ homomorphisms. Consider $n$ of the given homomorphism which we denote now, without distinction of isomorphism type, by $\left(\phi^{k}, \psi^{k}\right) \in \operatorname{Hom}\left(S^{k}, T^{k} ; V, W\right), 1 \leqq k \leqq n$. We assume also that the notation has been chosen so that if the systems $\left(S^{k}, T^{k}\right)$ are not all distinct, then the systems $\left(S^{k}, T^{k}\right), k=1, \cdots, m(2 \leqq m \leqq n)$, coincide and are different from the rest. Let $(\mu, v)$ be the natural homomorphism of $(V, W)$ onto

$$
(V, W) /\left(\phi^{1}, \psi^{1}\right)\left(S^{1}, T^{1}\right) .
$$

We show that the homomorphisms $\left(\tilde{\phi}^{k}, \tilde{\psi}^{k}\right)$ of $\left(S^{k}, T^{k}\right)$ into $(V, W) /\left(\phi^{1}, \psi^{1}\right)\left(S^{1}, T^{1}\right)$ defined by $\left(\tilde{\phi}^{k}, \tilde{\psi}^{k}\right)=(\mu, v)\left(\phi^{k}, \psi^{k}\right), \quad k=2, \cdots, n$, satisfy the hypotheses of the theorem. Let $K \subseteq\{2, \cdots, n\}$ be a maximal subset of indices for which the systems $\left(S^{k}, T^{k}\right), k \in K$ coincide, and let us denote this common system by $(S, T)$. Let $\left(\mu^{k}, v^{k}\right), k \in K$, be endomorphisms of $(S, T)$ at least one of which is an automorphism. We have to show that $(\tilde{\phi}, \tilde{\psi})=\sum_{k \in K}\left(\tilde{\phi}^{k}, \tilde{\psi}^{k}\right)\left(\mu^{k}, v^{k}\right)$ is a q. s. monomorphism. In case $(S, T)=\left(S^{1}, T^{1}\right)$ denote $(\phi, \psi)=\sum_{k \in K}\left(\phi^{k}, \psi^{k}\right)\left(\mu^{k}, v^{k}\right)$ and let $\left(\pi^{1}, \rho^{1}\right),(\pi, \rho)$ be endomorphisms of $(S, T)$ at least one of which is an automorphism. Then 
$\left(^{*}\right)\left(\phi^{1}, \psi^{1}\right)\left(\pi^{1}, \rho^{1}\right)+(\phi, \psi)(\pi, \rho)=\left(\phi^{1}, \psi^{1}\right)\left(\pi^{1}, \rho^{1}\right)+\sum_{k \in K}\left(\phi^{k}, \psi^{k}\right)\left(\left(\mu^{k}, v^{k}\right)(\pi, \rho)\right)$

is a combination of the elements of $\left\{\left(\phi^{1}, \psi^{1}\right)\right\} \cup\left\{\left(\phi^{k}, \psi^{k}\right) \mid k \in K\right\}$ with at least one automorphism as a coefficient. Hence, by our assumptions, $\left({ }^{*}\right)$ is a q.s. monomorphism. The case of two homomorphisms therefore implies that $\left(\phi^{1}, \psi^{1}\right)(S, T)+(\phi, \psi)(S, T)$ is a q.s. direct sum. The independence of these subsystems together with the fact that $(\phi, \psi)$ is a monomorphism imply that $(\tilde{\phi}, \tilde{\psi})=(\mu, v)(\phi, \psi)$ is a monomorphism. Since

$$
(\tilde{\phi}, \tilde{\psi})(S, T)=\left(\left(\phi^{1}, \psi^{1}\right)(S, T)+(\phi, \psi)(S, T)\right) /\left(\phi^{1}, \psi^{1}\right)(S, T),
$$

it follows from 1.3 that $(\tilde{\phi}, \tilde{\psi})$ is q.s. We have chosen the notation so that in case $(S, T) \neq\left(S^{1}, T^{1}\right)$ the set $\{1\} \cup K$ contains less than $n$ elements. Hence by our induction hypothesis $\left(\phi^{1}, \psi^{1}\right)\left(S^{1}, T^{1}\right)+\sum_{k \in K}\left(\phi^{k}, \psi^{k}\right)(S, T)$ is a q.s. direct sum. It follows from 1.3 and 1.4 that

$$
\sum_{k \in K}\left(\tilde{\phi}^{k}, \tilde{\psi}^{k}\right)(S, T)=\sum_{k \in K}\left(\left(\phi^{1}, \psi^{1}\right)(S, T)+\left(\phi^{k}, \psi^{k}\right)\left(S^{k}, T^{k}\right)\right) /\left(\phi^{1}, \psi^{1}\right)\left(S^{1}, T^{1}\right)
$$

is a q.s. direct sum. Therefore, by $4.1,(\tilde{\phi}, \tilde{\psi})$ is a q.s. monomorphism. It follows now from our induction hypothesis that

$$
\sum_{k=2}^{n}\left(\tilde{\phi}^{k}, \tilde{\psi}^{k}\right)\left(S^{k}, T^{k}\right)=\sum_{k=2}^{n}\left(\left(\phi^{1}, \psi^{1}\right)\left(S^{1}, T^{1}\right) \dot{+}\left(\phi^{k}, \psi^{k}\right)\left(S^{k}, T^{k}\right)\right) /\left(\phi^{1}, \psi^{1}\right)\left(S^{1}, T^{1}\right)
$$

is a direct sum which is q.s. in $(V, W) /\left(\phi^{1}, \psi^{1}\right)\left(S^{1}, T^{1}\right)$. Hence, again by 1.4 , and 1.3, we see that $\sum_{k=1}^{n}\left(\phi^{k}, \psi^{k}\right)\left(S^{k}, T^{k}\right)$ is a direct sum which is q.s. in $(V, W)$.

The condition of the last theorem for one system $(S, T)$ is always satisfied in case $(S, T)$ is algebraically compact (this result will not be used):

4.3. THEOREM. Let $\left(\phi_{1}, \psi_{1}\right),\left(\phi_{2}, \psi_{2}\right)$ be homomorphisms of an algebraically compact system $(S, T)$ into a system $(V, W)$ which are linearly independent modulo $D(S, T ; V, W)$. Then $\left(\phi_{1}, \psi_{2}\right),\left(\phi_{2}, \psi_{1}\right)$ are monomorphisms and $\left(\phi_{1}, \psi_{1}\right)(S, T)+\left(\phi_{2}, \psi_{2}\right)(S, T)$ is a direct sum which is q.s. in $(V, W)$.

Proof. Obviously $\left(\phi_{1}, \psi_{1}\right)$ and $\left(\phi_{2}, \psi_{2}\right)$ are q.s. monomorphisms. Hence $\left(\phi_{1}, \psi_{1}\right)(S, T)$ is an algebraically compact q.s. subsystem of $(V, W)$ and there exists a projection $(\pi, \rho)$ of $(V, W)$ onto $\left(\phi_{1}, \psi_{1}\right)(S, T)$. The pair of mappings $(\mu, v)=\left(\phi_{1}, \psi_{1}\right)^{-1}(\pi, \rho)\left(\phi_{2}, \psi_{2}\right)$ is a well-defined endomorphism of $(S, T)$. By the linear independence condition,

$$
\left(\phi_{2}, \psi_{2}\right)-\left(\phi_{1}, \psi_{1}\right)(\mu, v)=(1-\pi, 1-\rho)\left(\phi_{2}, \psi_{2}\right)
$$

is a q.s. monomorphism. This implies that $\left(\phi_{1}, \psi_{1}\right)(S, T) \cap\left(\phi_{2}, \psi_{2}\right)(S, T)=(0,0)$ and that $(1-\pi, 1-\rho)\left(\phi_{2}, \psi_{2}\right)(S, T)$ is q.s., and hence spectral, in $(1-\pi, 1-\rho)(V, W)$. Hence there exists a decomposition $(1-\pi, 1-\rho)(V, W)$ $=(1-\pi, 1-\rho)\left(\phi_{2}, \psi_{2}\right)(S, T)+(X, Y)$. Therefore $(V, W)=\left(\phi_{1}, \psi_{1}\right)(S, T)$ 
$\dot{+}(1-\pi, 1-\rho)(V, W)=\left(\phi_{1}, \psi_{1}\right)(S, T) \dot{+}(1-\pi, 1-\rho)\left(\phi_{2}, \psi_{2}\right)(S, T) !+(X, Y)$ and $\left(\phi_{1}, \psi_{1}\right)(S, T)+(1-\pi, 1-\rho)\left(\phi_{2}, \psi_{2}\right)(S, T)$ is spectral in $(V, W)$. Now $\left(^{*}\right)$ implies that $(1-\pi, 1-\rho)\left(\phi_{2}, \psi_{2}\right)(S, T) \subseteq\left(\phi_{1}, \psi_{1}\right)(S, T)+\left(\phi_{2}, \psi_{2}\right)(S, T)$ and $\left(\phi_{2}, \psi_{2}\right)$ $=\left(\phi_{1}, \psi_{1}\right)(\mu, v)+(1-\pi, 1-\rho)\left(\phi_{2}, \psi_{2}\right)$ implies that $\left(\phi_{2}, \psi_{2}\right)(S, T) \subseteq\left(\phi_{1}, \psi_{1}\right)(S, T)$ $+(1-\pi, 1-\rho)\left(\phi_{2}, \psi_{2}\right)(S, T)$. Hence $\left(\phi_{1}, \psi_{1}\right)(S, T)+\left(\phi_{2}, \psi_{2}\right)(S, T)=\left(\phi_{1}, \psi_{1}\right)(S, T)$ $+(1-\pi, 1-\rho)\left(\phi_{2}, \psi_{2}\right)(S, T)$ is q.s. in $:(V, W)$. We remark that by a slight modification in the argument the theorem may be proved for $(S, T)$ of finite dimension without making use of the fact that $(S, T)$ is algebraically compact.

In [1] the conclusion of Theorem 4.2. was proved for the family of all q.s.irr. types which are of finite dimension or torsion type, namely $\mathrm{I}_{m}, \mathrm{II}_{m}^{\theta}, \mathrm{III}_{m}$ and $\mathrm{II}_{\infty}^{\boldsymbol{\theta}}$. As we mentioned in 1.5, these types are algebraically compact (the proof of this fact for the divisible types $\mathrm{II}_{\infty}{ }^{\theta}$ relied on 1.13 which in turn involved the knowledge of $D(S, T ; V, W)$ ). For these types there was no need to consider the module $\operatorname{Hom}(S, T ; V, W)$. The homomorphisms were replaced by a vector space of chains, those corresponding to elements of $D(S, T ; V, W)$ forming an explicitly described subspace. Instead of linear dependence modulo $D(S, T ; V, W)$, the conditions were expressed by linear dependence of elements in the quotient space. This was possible because for these types $D(S, T ; V, W)$ is a submodule of $\operatorname{Hom}(S, T ; V, W)$ and in addition the endomorphisms are sums of scalar multiples of the identity and endomorphisms which are not monomorphisms (for $\mathrm{I}_{m}, \mathrm{III}_{m}$ just scalar multiples of the identity). We included 4.3 to indicate the way in which some of the results of [1] could be reestablished. More detailed statements are not hard to obtain for $(S, T)$ q.s.irr. and algebraically compact. However, since we do not have new examples of such isomorphism types (see 5.8) we feel that their further discussion is warranted only in a more general setting.

Returning to general $(S, T)$, we define the relation $\prec$ between elements and subsets of $\operatorname{Hom}(S, T ; V, W)$ as follows: $(\phi, \psi) \prec L$ means that there exist finite subsets $\left\{\left(\phi_{k}, \psi_{k}\right) \mid k=1, \cdots, n\right\} \subseteq L$ and $\left\{\left(\mu_{k}, v_{k}\right) \mid k=1, \cdots, n\right\} \subseteq \operatorname{End}(S, T)$ such that

$$
(\phi, \psi)-\sum_{k=1}^{n}\left(\phi_{k}, \psi_{k}\right)\left(\mu_{k}, v_{k}\right) \in D(S, T ; V, W) .
$$

No confusion with the dependence relation introduced in $\$ 2$ will arise. Evidently a subset $M$ of $\operatorname{Hom}(S, T ; V, W)$ is linearly dependent modulo $D(S, T ; V, W)$ if and only if there exists an element $(\phi, \psi)$ in $M$ such that $(\phi, \psi) \prec M-\{(\phi, \psi)\}$. It is immediate that for $(S, T) \neq(0,0)$ the relation $\prec$ satisfies the dependence postulates (b), (c), (d) of Theorem 2.3 and that the case $n=1$ of the exchange postulate (e) is sufficient for its general validity. Hence, by the proof of 4.3 and by 4.1 , the exchange postulate holds for $(S, T)$ algebraically compact. However, there are systems $(S, T)$, even q.s.irr. ones, for which $D(S, T ; V, W)$ is not closed under addition (see next section). Then, if $\left(\phi_{1}, \psi_{1}\right),\left(\phi_{2}, \psi_{2}\right) \in D(S, T ; V, W)$ while $\left(\phi_{1}, \psi_{1}\right)+\left(\phi_{2}, \psi_{2}\right)$ is a q.s. monomorphism, we have 


$$
\left(\phi_{1}, \psi_{1}\right)+\left(\phi_{2}, \psi_{2}\right) \prec\left\{\left(\phi_{1}, \psi_{1}\right)\right\},\left(\phi_{1}, \psi_{1}\right) \prec \varnothing
$$

and $\left(\phi_{1}, \psi_{1}\right)+\left(\phi_{2}, \psi_{2}\right)$ not $\prec \varnothing$; so that the transitivity postulate (a) of 2.3 is not satisfied. It would be interesting to know whether even in this case one can define a quotient object of $\operatorname{Hom}(S, T ; V, W)$ modulo $D(S, T ; V, W)$ which will yield a significant invariant of $(V, W)$ corresponding to the isomorphism type of $(S, T)$. We do not pursue this problem here except to note that $D(S, T ; V, W)$ is closed under scalar multiplication:

4.4. THEOREM. Let $(S, T)$ be a q.s.irr. system and $(V, W)$ an arbitrary system. If $(\phi, \psi) \in D(S, T ; V, W)$ and $(\mu, v) \in \operatorname{End}(S, T)$, then $(\phi, \psi)(\mu, v) \in D(S, T ; V, W)$.

Proof. Suppose conversely that $(\phi, \psi)(\mu, v)$ is a q.s. monomorphism. Then if $\left(S^{\prime}, T^{\prime}\right)$ is a finite-dimensional subsystem of $(S, T)$, there exists a decomposition

$$
(\phi, \psi)(\mu, v)(S, T)+(\phi, \psi)\left(S^{\prime}, T^{\prime}\right)=(\phi, \psi)(\mu, v)(S, T)+(X, Y)
$$

where $(X, Y) \subseteq(\phi, \psi)(S, T)$. Even if $(\phi, \psi)$ is not a monomorphism, $\left(\phi^{-1} X, \psi^{-1} Y\right)$ is a well-defined subsystem of $\left(S, T^{\prime}\right)$. Using the fact that $(\phi, \psi)(\mu, v)$ is a monomorphism, one readily checks that

$$
(\mu, v)(S, T)+\left(S^{\prime}, T^{\prime}\right)=(\mu, v)(S, T)+\left(\phi^{-1} X, \psi^{-1} Y\right) \cap\left((\mu, v)(S, T)+\left(S^{\prime}, T^{\prime}\right)\right) .
$$

Hence $(\mu, v)(S, T)$ is q.s. in $(S, T)$. Since $(\mu, v)$ is clearly a monomorphism and since $(S, T)$ is q.s.irr., we have $(\mu, v)(S, T) \neq(0,0)$ and therefore $(\mu, v)(S, T)=(S, T)$. Hence $(\mu, v)$ is an automorphism, and from this the contradiction that $(\phi, \psi)$ is a q.s. monomorphism follows immediately.

\section{Quasi-spectral direct sums of torsion-free systems of rank 1.}

5.1. LemMA. Let $(X, Y)$ be a torsion-free q.s. subsystem of $(V, W)$ such that $(V, W) /(X, Y)$ is of type $\mathrm{II}_{\infty}^{\theta}$. Then $(X, Y)$ is spectral in $(V, W)$.

Proof. Let $U$ be a direct complement of $X$ in $V: V=X+U$. Let $\left\{u_{k}\right\}$ be a sequence of elements of $U$ representing a chain in $C_{-\infty}^{-\infty}{ }_{0}^{\infty}\left(A, B_{\theta} ; V / X, W / Y\right)$ which spans $(V, W) /(X, Y)$. Since $(X, Y)$ is q.s. in $(V, W)$, for every integer $m \geqq 0$ there exists a projection $\left(\pi_{m}, \rho_{m}\right)$ of $\left(V_{m}, W_{m}\right)=\left(X+\left[u_{-m}, \cdots, u_{0}\right], Y+\mathscr{T}\left[u_{-m}, \cdots, u_{0}\right]\right)$ onto $(X, Y)$. By changing if necessary the roles of $A$ and $B$ we may assume that $\theta \neq \infty$. Operating on $B_{\theta} u_{0} \in Y, A u_{k}-B_{\theta} u_{k-1} \in Y,-m<k \leqq 0$, with $1-\rho_{m}$, we obtain $B_{\theta}\left(1-\pi_{m}\right) u_{0}=0, A\left(1-\pi_{m}\right) u_{k}-B_{\theta}\left(1-\pi_{m}\right) u_{k-1}=0,-m<k \leqq 0$. Thus $\left\{\left(1-\pi_{m}\right) u_{k}\right\}_{-m}^{\infty} \in C_{-m 0}^{-m \infty}\left(A, B_{\theta} ; V, W\right)$ (evidently $u_{k}=0, k \geqq 1$ ). Hence if $n>m$, we have

$$
\left\{\left(\pi_{n}-\pi_{m}\right) u_{k}\right\}_{-m}^{\infty} \in C_{-m}^{-m}{ }_{0}^{\infty}\left(A, B_{\theta} ; X, Y\right) .
$$

Since $(X, Y)$ is torsion-free, the last chain vanishes identically, so that $\pi_{m}$ is the restriction of $\pi_{n}$ to $V_{m}$. From the relations $\rho_{n} C v=C \pi_{n} v=C \pi_{m} v=\rho_{m} C v$, where 
$C$ is $A$ or $B_{\theta}$ and $v \in V_{m}$, it follows that $\rho_{m}$ is the restriction of $\rho_{n}$ to $W_{m}$. Hence there exists a pair $(\pi, \rho)$ of linear transformations of $V$ and $W$ into themselves which for every $m \geqq 0$ coincides with $\left(\pi_{m}, \rho_{m}\right)$ on $\left(V_{m}, W_{m}\right)$. Since $\mathscr{T}(V / X)=W / Y$, we have $W=Y+\mathscr{T} U$. Thus $(V, W)=\bigcup_{m=0}^{\infty}\left(V_{m}, W_{m}\right)$, and $(\pi, \rho)$ is a projection of $(V, W)$ onto $(X, Y)$.

5.2. THEOREM. Let $(X, Y)$ be a torsion-free q.s. subsystem of $(V, W)$ such that $(V, W) /(X, Y)$ is a torsion system. Then $(X, Y)$ is spectral in $(V, W)$.

Proof. The deduction of this theorem from 1.13 and 5.1 follows Sasiada's characterization of algebraically compact abelian groups (see [4, p. 83]). The argument was used in [1] in showing that a divisible system is algebraically compact. We include it here for the sake of completeness. Let $\left\{\left(V_{\alpha}, W_{\alpha}\right) \mid 0 \leqq \alpha \leqq \lambda\right\}$ be a transfinite sequence of subsystems of $(V, W) /(X, Y)$ which has the properties of 1.13 relative to the torsion system $(V, W) /(X, Y)$ (instead of relative to $(V, W)$ ). We set $\left(V_{\alpha}, W_{\alpha}\right)=\left(X_{\alpha}, Y_{\alpha}\right) /(X, Y)$ and construct by transfinite induction an increasing sequence $\left\{\left(U_{\alpha}, Z_{\alpha}\right) \mid 0 \leqq \alpha \leqq \lambda\right\}$ of subsystems of $(V, W)$ such that

$$
\left(X_{\alpha}, Y_{\alpha}\right)=(X, Y)+\left(U_{\alpha}, Z_{\alpha}\right) \text {. }
$$

Then $\left(U_{\lambda}, Z_{\lambda}\right)$ will be a direct complement of $(X, Y)$ in $(V, W)$. We take $\left(U_{0}, Z_{0}\right)=(0,0)$. Suppose that the subsystems $\left(U_{\alpha}, Z_{\alpha}\right)$ are already defined for $\alpha<\beta(\beta \leqq \lambda)$. If $\beta$ is a limit ordinal, we define $\left(U_{\beta}, Z_{\beta}\right)=\bigcup_{\alpha<\beta}\left(U_{\alpha}, Z_{\alpha}\right)$. If $\beta-1$ exists, then, being isomorphic to $(X, Y),\left(X_{\beta-1}, Y_{\beta-1}\right) /\left(U_{\beta-1}, Z_{\beta-1}\right)$ is torsion free. Since $\left(V_{\beta-1}, W_{\beta-1}\right)$ is q.s. in $(V, W) /(X, Y)$ and $(X, Y)$ is q.s. in $(V, W)$, it follows from 1.3 that $\left(X_{\beta-1}, Y_{\beta-1}\right)$ is q.s. in $(V, W)$ and hence $\left(X_{\beta-1}, Y_{\beta-1}\right) /\left(U_{\beta-1}, Z_{\beta-1}\right)$ is q.s. in $\left(X_{\beta}, Y_{\beta}\right) /\left(U_{\beta-1}, Z_{\beta-1}\right)$. The quotient system of the last two systems, which is isomorphic to $\left(V_{\beta}, W_{\beta}\right) /\left(V_{\beta-1}, W_{\beta-1}\right)$, is either of finite dimension or of some type $\mathrm{II}_{\infty}^{\theta}$. Applying Lemma 5.1 in the case of the second alternative, we obtain a decomposition

$$
\begin{array}{r}
\left(X_{\beta}, Y_{\beta}\right) /\left(U_{\beta-1}, Z_{\beta-1}\right)=\left(X_{\beta-1}, Y_{\beta-1}\right) /\left(U_{\beta-1}, Z_{\beta-1}\right)+\left(U_{\beta}, Z_{\beta}\right) /\left(U_{\beta-1}, Z_{\beta-1}\right), \\
\left(U_{\beta}, Z_{\beta}\right) \supset\left(U_{\beta-1}, Z_{\beta-1}\right) .
\end{array}
$$

This together with the case $\alpha=\beta-1$ of $\left(^{*}\right)$ implies that $\left(^{*}\right)$ holds for $\alpha=\beta$.

5.3. LEMMA. Let $(X, Y)$ be a torsion-free q.s. subsystem of $(V, W)$. Then $t(V, W) \cap(X, Y)=(0,0)$ and $t(V, W)+(X, Y)$ is q.s. in $(V, W)$.

Proof. Let $x \in X$ belong to the domain of $t(V, W)$. By 1.11 there exists a finitedimensional torsion subsystem $(U, Z)$ of $t(V, W)$ such that $x \in U$. Since $(X, Y)$ is q.s. in $(V, W)$, there exists a projection $(\pi, \rho)$ of $(X, Y)+(U, Z)$ onto $(X, Y)$. By $1.10,(\pi, \rho)(U, Z)$ is a torsion subsystem of the torsion-free system $(X, Y)$. Hence, 1.11 , it vanishes, and $x=\pi x=0$. The same argument is valid for the range spaces. Thus $t(V, W) \cap(X, Y)=(0,0)$. By $2 \cdot 1(\mathrm{~d})$ we have 


$$
\operatorname{cl}(X, Y) /(X, Y)=t((V, W) /(X, Y)) \text {. }
$$

According to 1.13 this is a q.s. subsystem of $(V, W) /(X, Y)$, and since $(X, Y)$ is q.s. in $(V, W)$, it follows from 1.3 that $\operatorname{cl}(X, Y)$ is q.s. in $(V, W)$. We complete the proof by showing that $\operatorname{cl}(X, Y)=t(V, W)+(X, Y)$. By 2.1(a)(c),

$$
\operatorname{cl}(X, Y) \supseteq \operatorname{cl}(\varnothing, \varnothing)=t(V, W), \quad \operatorname{cl}(X, Y) \supseteq(X, Y) ;
$$

thus $\operatorname{cl}(X, Y) \supseteq t(V, W)+(X, Y)$. On the other hand, Theorem 5.3 implies that $(X, Y)$ is spectral in $\operatorname{cl}(X, Y)$. A direct complement of $(X, Y)$ in $\operatorname{cl}(X, Y)$ is a torsion system, and hence, 1.12, included in $t(V, W)$. This proves the required reverse inclusion.

An immediate consequence of 5.3, 1.3 and 1.13 is

5.4. CoRollary. Let $(\phi, \psi)$ be a homomorphism of a torsion-free system $(S, T)$ into a system $(V, W)$, and let $(\mu, v)$ be the natural homomorphism of $(V, W)$ onto $(V, W) / t(V, W)$. Then $(\phi, \psi) \in D(S, T ; V, W)$ if and only if

$$
(\mu, v)(\phi, \psi) \in D(S, T ;(V, W) / t(V, W)) .
$$

We also have

5.5. Corollary. Let $\left(S_{1}, T_{1}\right)$ be a torsion system and let $\left(S_{2}, T_{2}\right)$ be torsionfree. If $\left(\phi_{i}, \psi_{i}\right), i=1,2$ are q.s. monomorphisms of $\left(S_{i}, T_{i}\right)$ respectively into a system $(V, W)$, then

$$
\left(\phi_{1}, \psi_{1}\right)\left(S_{1}, T_{1}\right)+\left(\phi_{2}, \psi_{2}\right)\left(S_{2}, T_{2}\right)
$$

is a direct sum which is q.s. in $(V, W)$.

Proof. By 1.12, $\left(\phi_{1}, \psi_{1}\right)\left(S_{1}, T_{1}\right) \subseteq t(V, W)$. From 5.3 the independence of the image systems follows immediately, while to prove that their sum is q.s. it suffices according to 1.1 to show that it is q.s. in $t(V, W)+\left(\phi_{2}, \psi_{2}\right)\left(S_{2}, T_{2}\right)$. Let $(X, Y)$ be a finite-dimensional subsystem of $t(V, W)+\left(\phi_{2}, \psi_{2}\right)\left(S_{2}, T_{2}\right)$, and let $\left(\pi_{i}, \rho_{i}\right), i=1,2$ denote the projections associated with the last decomposition. Then $\left(\phi_{1}, \psi_{1}\right)\left(S_{1}, T_{1}\right)+\left(\pi_{1}, \rho_{1}\right)(X, Y)$ is a finite-dimensional extension of $\left(\phi_{1}, \psi_{1}\right)\left(S_{1}, T_{1}\right)$, and hence there exists a decomposition

$\left(\phi_{1}, \psi_{1}\right)\left(S_{1}, T_{1}\right)+\left(\pi_{1}, \rho_{1}\right)(X, Y)=\left(\phi_{1}, \psi_{1}\right)\left(S_{1}, T_{1}\right)+\left(X^{\prime}, Y^{\prime}\right),\left(X,^{\prime} Y^{\prime}\right) \subseteq t(V, W)$.

Therefore

$$
\begin{aligned}
\left(\phi_{1}, \psi_{1}\right)\left(S_{1}, T_{1}\right)+( & \left.\phi_{2}, \psi_{2}\right)\left(S_{2}, T_{2}\right)+\left(\pi_{1}, \rho_{1}\right)(X, Y) \\
& \left.=\left(\phi_{1}, \psi_{1}\right)\left(S_{1}, T_{1}\right)+\left(\phi_{2}, \psi_{2}\right)\left(S_{2}, T_{2}\right)+X^{\prime}, Y^{\prime}\right) .
\end{aligned}
$$

Hence $\left(\phi_{1}, \psi_{1}\right)\left(S_{1}, T_{1}\right)+\left(\phi_{2}, \psi_{2}\right)\left(S_{2}, T_{2}\right)$ is spectral in the system $\left(^{*}\right)$. Since $\left(\pi_{2}, \rho_{2}\right)(X, Y) \subseteq\left(\phi_{2}, \psi_{2}\right)\left(S_{2}, T_{2}\right)$, the system $\left(^{*}\right)$ includes 


$$
\left(\phi_{1}, \psi_{1}\right)\left(S_{1}, T_{1}\right)+\left(\phi_{2}, \psi_{2}\right)\left(S_{2}, T_{2}\right)+(X, Y) .
$$

Therefore $\left(\phi_{1}, \psi_{1}\right)\left(S_{1}, T_{1}\right)+\left(\phi_{2}, \psi_{2}\right)\left(S_{2}, T_{2}\right)$ is spectral in $\left(\phi_{1}, \psi_{1}\right)\left(S_{1}, T_{1}\right)$ $+\left(\phi_{2}, \psi_{2}\right)\left(S_{2}, T_{2}\right)+(X, Y)$; i.e., it is q.s. in $t(V, W)+\left(\phi_{2}, \psi_{2}\right)\left(S_{2}, T_{2}\right)$.

Corollary 5.4 together with the next theorem yield a characterization of $D(S, T ; V, W)$ when $(S, T)$ is an infinite-dimensional torsion-free system of rank 1 .

5.6. THEOREM. Let $(X, Y)$ be an infinite-dimensional torsion-free system of rank 1 which is a torsion-closed subsystem of $(V, W)$. Then $(X, Y)$ is q.s. in $(V, W)$.

Proof. From the assumption that $(V, W) /(X, Y)$ is torsion-free and from 1.8 it follows that it will be sufficient to prove that $(X, Y)$ is spectral in subsystems $(U, Z)$ of $(V, W)$ such that $(U, Z) /(X, Y)$ is of type $\operatorname{III}_{m}$. Since every system is obviously spectral in extensions by type $\mathrm{III}_{-1}$ or $\mathrm{III}_{0}$, we suppose for the purpose of induction that $m \geqq 1$ and that the statement is true for extensions by type $\mathrm{III}_{m-1}$. From 1.6 one sees that $(U, Z) /(X, Y)$ contains a subsystem $\left(U^{\prime}, Z^{\prime}\right) /(X, Y)$ of type $\mathrm{III}_{m-1}$ such that

$$
(U, Z) /\left(U^{\prime}, Z^{\prime}\right) \cong((U, Z) /(X, Y)) /\left(\left(U^{\prime}, Z^{\prime}\right) /(X, Y)\right)
$$

is of type $\mathrm{II}_{0}^{\infty}$. This means that there exists an element $u \in U$ such that $(U, Z)=\left(U^{\prime}, Z^{\prime}\right)+([u], \mathscr{T}[u])$ and $A u \in Z^{\prime}$. By the induction hypothesis there is a decomposition $\left(U^{\prime}, Z^{\prime}\right)=(X, Y)+\left(U^{\prime \prime}, Z^{\prime \prime}\right)$. Then $A u=y+z^{\prime \prime}$, where $y \in Y, z^{\prime \prime} \in Z^{\prime \prime}$. The system $\left(U^{\prime \prime}, Z^{\prime \prime}\right)$ is of type $\mathrm{III}_{m-1}$; hence it is spanned by a chain $\left\{u_{k}^{\prime \prime}\right\}_{0}^{m-1} \in C_{0}^{0}{ }_{m-1}^{m-1}\left(A, B ; U^{\prime \prime}, Z^{\prime \prime}\right)$ with corresponding range elements $\left\{z_{k}^{\prime \prime}\right\}_{0}^{m}$. From the assumptions on $(X, Y)$ and from Lemma 2.2 it follows that $(X, Y)$ contains a subsystem $\left(X^{\prime}, Y^{\prime}\right)$ of type $\operatorname{III}_{n}, n \geqq m-1$, such that $y \in Y^{\prime}$. Let $\left\{x_{k}^{\prime}\right\}_{0}^{n}$ be a chain in $C_{0}^{0}{ }_{n}^{n}\left(A, B ; X^{\prime}, Y^{\prime}\right)$ with range elements $\left\{y_{k}^{\prime}\right\}_{0}^{n+1}$ which spans $\left(X^{\prime}, Y^{\prime}\right)$. We then have representations $z^{\prime \prime}=\sum_{k=0}^{m} \alpha_{k} z_{k}^{\prime \prime}, y=\sum_{k=0}^{n+1} \beta_{k} y_{k}^{\prime \prime}$. It is easy to verify that the sequence

$$
\begin{aligned}
\left(\alpha_{m} u_{0}^{\prime \prime}+\beta_{n+1} x_{n-m+1}^{\prime}, \alpha_{m} u_{1}^{\prime \prime}+\beta_{n+1} x_{n-m+2}^{\prime}, \cdots, \alpha_{m} u_{m-1}^{\prime \prime}\right. & \\
& \left.+\beta_{n+1} x_{n}^{\prime}, u-\sum_{k=0}^{m-1} \alpha_{k} u_{k}^{\prime \prime}-\sum_{k=0}^{n} \beta_{k} x_{k}^{\prime}\right)
\end{aligned}
$$

is a chain in $C_{0}^{0}{ }_{m}^{m}(A, B ; U, Z)$ with range elements

$$
\begin{aligned}
\left(\alpha_{m} z_{0}^{\prime \prime}+\beta_{n+1} y_{n-m+1}^{\prime},\right. & \alpha_{m} z_{1}^{\prime \prime}+\beta_{n+1} y_{n-m+2}^{\prime}, \cdots, \alpha_{m} z_{m}^{\prime \prime} \\
& \left.+\beta_{n+1} y_{n+1}^{\prime}, B u-\sum_{k=0}^{m-1} \alpha_{k} z_{k+1}^{\prime \prime}-\sum_{k=0}^{n} \beta_{k} y_{k+1}^{\prime}\right) .
\end{aligned}
$$

We have $\alpha_{m} \neq 0$ since otherwise the last element of $\left(^{*}\right)$, which is congruent modulo $U^{\prime}$ to $u$ and therefore does not belong to $U^{\prime}$ or $X$, would be transformed by $A$ into $\beta_{n+1} y_{n+1}^{\prime} \in Y$. This would be against the fact that $(V, W) /(X, Y)$ is 
torsion-free. Hence reducing the sequences $\left({ }^{*}\right)$ and $\left({ }^{* *}\right)$ modulo $X^{\prime}$ and $Y^{\prime}$ respectively one sees that they are linearly independent modulo $X$ and $Y$ respectively. Therefore they span a subsystem of type $\operatorname{III}_{m}$ independent of $(X, Y)$; i.e., a direct complement of $(X, Y)$ in $(U, Z)$.

We remark that a proof of the last theorem could have been based on the fact that systems of type $\mathrm{III}_{n}$ are spectral in extensions by a system of type $\mathrm{III}_{m}$ if $n \geqq m$. This is a corollary of the explicit determination obtained in [1] of $D(S, T ; V, W)$ for $(S, T)$ of type $\mathrm{III}_{n}$. However the restriction $n \geqq m$ imposed here is necessary and therefore 5.6 does not hold for $(X, Y)$ of finite dimension (cf. the following example or 5.8).

At this point the reader may wonder whether there exist q.s.irr. torsion-free systems which are not of rank 1. It follows from Theorem 5.6 that in such a system every nonzero range element must generate a finite-dimensional system (i.e. of some type $\mathrm{III}_{m}$ ). In particular such a system must be singular (it is easy to see that every indecomposable torsion-free module over the complex polynomials gives rise through the correspondence mentioned in $\$ 1$ to a nonsingular indecomposable torsion-free system of the same rank). We shall be content with the following example (cf. the example of an indecomposable torsion-free module of rank 2 in Kaplansky [6]).

5.7. EXAmple. There exists a q.s.irr. torsion-free system of rank 2 .

Let $V$ be the vector space of all the polynomials in an indeterminate $\zeta$ over the complex field, and let $W$ be of the form $W=V+\left[w_{0}\right]$, where $w_{0} \neq 0$. We make $(V, W)$ into a system by requiring for a fixed basis $A, B$ of $\mathscr{T}$ the following relations

$$
\begin{aligned}
& A \zeta^{k}=\zeta^{k}+\alpha_{k} w_{0}, \\
& B \zeta^{k}=\zeta^{k+1}, \quad k=0,1,2, \cdots,
\end{aligned}
$$

where the scalars $\alpha_{k}$ are chosen so that the formal power series $\sum_{k=0}^{\infty} \alpha_{k} \zeta^{k}$ is not the expansion of a rational function of $\zeta$. The quotient system $(V, W) /\left(0,\left[w_{0}\right]\right)$ is isomorphic to the torsion-free system of rank $1(S, T)_{A, B}^{H}$, where $H_{\theta}=0$ for $\theta \neq \infty$ and $H_{\infty}=\infty$ (this notation was introduced at the end of the proof of 3.4). It follows from Theorem 2.4 that $(V, W)$ is of rank 2, and of course torsion-free. Any nontrivial q.s. subsystem $(X, Y)$ of $(V, W)$ must, by the same theorem, be of rank 1 . If $(X, Y)$ itself is not of infinite dimension, it has according to 1.5 a direct complement which is of infinite dimension (and rank 1). In either case this implies the existence of an element $w \neq 0$ in $W$ such that $\sum_{\theta \in \Theta} H(w)_{\theta}=\infty$ (the heights may be considered in $(V, W)$ ). Since $\left(0,\left[w_{0}\right]\right)$ is torsion-closed in $(V, W)$, the height function of $w_{0}$ in $(V, W)$ is the same as in $\left(0,\left[w_{0}\right]\right)$. Therefore $w$ is not a scalar multiple of $w_{0}$. The sum of the heights of $w$ at the finite points $\theta$ does not exceed the corresponding sum for the image of $w$ in $(V, W) /\left(0,\left[w_{0}\right]\right)$, and the latter is finite by 3.4 . Thus $H(w)_{\infty}=\infty$. We show 
that this is impossible. Suppose $w=p(\zeta)+\beta w_{0}=\sum_{k=0}^{n} \beta_{k} \zeta^{k}+\beta w_{0}, p(\zeta) \neq 0$. In order that $A q(\zeta)=p(\zeta)+\beta w_{0}$, we must have $q(\zeta)=p(\zeta)$ and $\sum_{k=0}^{n} \beta_{k} \alpha_{k}=0$, and then $(B / A) w=\zeta p(\zeta)$. The applicability of higher powers of $B / A$ implies similarly the relations $\sum_{k=0}^{n} \beta_{k} \alpha_{k+m}=0, m=1,2, \cdots$. But this means that $\sum_{k=0}^{\infty} \alpha_{k} \zeta^{k}$ is an expansion of a rational function, against the choice of the $\alpha_{k}$ 's.

We shall show below that the conditions of Theorem 4.2 are partially satisfied for torsion-free systems of rank 1 . Theorem 4.3 is not applicable here:

5.8. THEOREM. A torsion-free system of rank 1 which is not of type $\mathrm{III}_{m}$ or $\mathscr{R}$ is not algebraically compact.

Proof. By an appropriate choice of a basis $A, B$ of $\mathscr{T}$ we may assume that the system in question, which is of infinite dimension, is $(S, T)_{A, B}^{H}$ where $H_{\infty}<\infty$. Let the vector spaces $V, W$ be of the form

$$
\begin{array}{ll}
V=S_{A, B}^{H}+\sum_{k=0}^{\infty}+\left[v_{k}\right], & v_{k} \neq 0, \\
W=T_{A, B}^{H}+\sum_{k=0}^{\infty}+\left[w_{k}\right], & w_{k} \neq 0 .
\end{array}
$$

We define a system operation in $(V, W)$ by the requirements that $(S, T)_{A, B}^{H}$ be a subsystem of $(V, W)$ and that

$$
\begin{aligned}
& A v_{k}=w_{k}, \\
& B v_{k}=w_{k+1}+1 \quad\left(\text { here } 1 \in T_{A, B}^{H}\right), \quad k=0,1,2, \cdots .
\end{aligned}
$$

It is clear that $(V, W) /(S, T)_{A, B}^{H}$ is torsion-free (of the same type as in the former example). Hence, by 5.6, $(S, T)_{A, B}^{H}$ is q.s. in $(V, W)$. On the other hand, if $(\pi, \rho)$ were a projection of $(V, W)$ onto $(S, T)_{A, B}^{H}$, we would have the relations

$$
\begin{aligned}
\zeta \cdot\left(\rho w_{k}\right) & =\zeta \cdot\left(\rho A v_{k}\right)=\zeta \cdot\left(A \pi v_{k}\right)=\zeta \cdot\left(\pi v_{k}\right)=B \pi v_{k} \\
& =\rho B v_{k}=\rho\left(w_{k+1}+1\right)=\rho w_{k+1}+1, \quad k=0,1,2, \cdots .
\end{aligned}
$$

From these it would follow recursively that $\rho w_{k}=\zeta^{k} \cdot \rho w_{0}-\sum_{j=0}^{k-1} \zeta^{j}$. But then $-\mathscr{W}\left(\rho w_{k}\right)_{\infty}$ would attain arbitrarily large values, against the assumption that $H_{\infty}<\infty$.

5.9. Lemma. Let $\left(\phi_{1}, \psi_{1}\right),\left(\phi_{2}, \psi_{2}\right)$ be homomorphisms of a torsion-free system of rank $1(S, T)$ into a system $(V, W)$ which are linearly independent modulo $D(S, T ; V, W)$. Then $\left(\phi_{1}, \psi_{1}\right),\left(\phi_{2}, \psi_{2}\right)$ are monomorphisms and $\left(\phi_{1}, \psi_{1}\right)(S, T)+\left(\phi_{2}, \psi_{2}\right)(S, T)$ is a direct sum which is q.s. in $(V, W)$.

Proof. Obviously $\left(\phi_{1}, \psi_{1}\right)$ and $\left(\phi_{2}, \psi_{2}\right)$ are q.s. monomorphisms. If we had $\left(\phi_{1}, \psi_{1}\right)(S, T) \cap\left(\phi_{2}, \psi_{2}\right)(S, T) \neq(0,0)$, then 


$$
\begin{aligned}
\left(\left(\phi_{1}, \psi_{1}\right)\right. & \left.(S, T)+\left(\phi_{2}, \psi_{2}\right)(S, T)\right) /\left(\phi_{1}, \psi_{1}\right)(S, T) \\
& \cong\left(\phi_{2}, \psi_{2}\right)(S, T) /\left(\left(\phi_{1}, \psi_{1}\right)(S, T) \cap\left(\phi_{2}, \psi_{2}\right)(S, T)\right)
\end{aligned}
$$

would be, by 2.2 , a torsion system. This would imply, according to 5.2 , the existence of a projection $(\pi, \rho)$ of $\left(\phi_{1}, \psi_{1}\right)(S, T)+\left(\phi_{2}, \psi_{2}\right)(S, T)$ onto $\left(\phi_{1}, \psi_{1}\right)(S, T)$. But then, by the argument used in the proof of 4.3 , we would get $\left(\phi_{1}, \psi_{1}\right)(S, T) \cap\left(\phi_{2}, \psi_{2}\right)(S, T)=(0,0)$. This contradiction shows that

$$
(X, Y)=\left(\phi_{1}, \psi_{1}\right)(S, T)+\left(\phi_{2}, \psi_{2}\right)(S, T)
$$

is a direct sum. To show that $(X, Y)$ is also q.s., we may, by 1.5 and 4.3 (or by referring to [1]), restrict ourselves to the case that $(S, T)$ is of infinite dimension. We assume without loss of generality that $(S, T)=(S, T)_{A, B}^{H}$ for some height function $H$. We also assume first that $(V, W)$ is torsion-free. Under this assumption we shall show that $(X, Y)$ is torsion-closed in $(V, W)$. It will follow that $(X, Y) /\left(\phi_{1}, \psi_{1}\right)(S, T)$, which by the directness of the sum is isomorphic to $(S, T)$, is torsion-closed in $(V, W) /\left(\phi_{1}, \psi_{1}\right)(S, T)$. Theorem 5.6 will then imply that $(X, Y) /\left(\phi_{1}, \psi_{1}\right)(S, T)$ is q.s. in $(V, W) /\left(\phi_{1}, \psi_{1}\right)(S, T)$. Hence, by $1.3,(X, Y)$ is q.s. in $(V, W)$. Let $v \in V, \theta \in \Theta$ be such that $B_{\theta} v \in Y$. Then $B_{\theta} v=\psi_{1} t_{1}+\psi_{2} t_{2}$, where $t_{1}, t_{2} \in T$. If $H_{\theta}=\infty$, then $H\left(t_{1}\right)_{\theta}=H\left(t_{2}\right)_{\theta}=\infty$, and there exist $s_{i} \in S$ such that $B_{\theta} s_{i}=t_{i}, i=1,2$. Then

$$
B_{\theta}\left(v-\phi_{1} s_{1}-\phi_{2} s_{2}\right)=0,
$$

and since $(V, W)$ is torsion-free, $v=\phi_{1} s_{1}+\phi_{2} s_{2} \in X$. Suppose now that $H_{\theta}<\infty$. It follows from (3.4.1) that we can represent $t_{i}, i=1,2$, in the form

$$
t_{i}(\zeta)=z_{i}(\zeta)+\alpha_{i}(\zeta-\theta)^{-H_{\theta}}
$$

(or $t_{i}(\zeta)=z_{i}(\zeta)+\alpha_{i} \zeta^{H_{\infty}}$ if $\theta=\infty$ ), where $\alpha_{i}$ is a scalar and $-\mathscr{W}\left(z_{i}\right)_{\theta} \leqq H_{\theta}-1$. Denote $s_{i}(\zeta)=z_{i}(\zeta) /(\zeta-\theta)\left(\right.$ or $s_{i}(\zeta)=z_{i}(\zeta)$ if $\left.\theta=\infty\right)$. Then (if $\left.\theta \neq \infty\right)$

$$
-\mathscr{W}\left(s_{i}\right)_{\eta}=-\mathscr{W}\left(z_{i}\right)_{\eta}+\mathscr{W}(z-\theta)= \begin{cases}-\mathscr{W}\left(z_{i}\right)_{\eta} \leqq H_{\eta} & \text { for } \eta \neq \theta, \infty, \\ -\mathscr{W}\left(z_{i}\right)_{\theta}+1 \leqq H_{\theta} & \text { for } \eta=\theta, \\ -\mathscr{W}\left(z_{i}\right)_{\infty}-1 \leqq H_{\infty}-1 & \text { for } \eta=\infty\end{cases}
$$

Thus by (3.4.2), $s_{i} \in S$ (a similar check sustains this conclusion in case $\theta=\infty$ ). We then have

$$
B_{\theta}\left(v-\phi_{1} s_{1}-\phi_{2} s_{2}\right)=\psi_{1} t_{1}+\psi_{2} t_{2}-\psi_{1} z_{1}-\psi_{2} z_{2}=\left(\alpha_{1} \psi_{1}+\alpha_{2} \psi_{2}\right) t,
$$

where $t=(\zeta-\theta)^{-H_{\theta}}$ (or $\zeta^{H_{\infty}}$ ) belongs to $T$. If $\alpha_{1}=\alpha_{2}=0$, then $\left({ }^{* *}\right)$ reduces to $\left(^{*}\right)$, and therefore $v \in X$. Otherwise it follows from the assumption of linear independence modulo $D(S, T ; V, W)$ that $(\phi, \psi)=\alpha_{1}\left(\phi_{1}, \psi_{1}\right)+\alpha_{2}\left(\phi_{2}, \psi_{2}\right)$ is q.s. and hence, by $2.1(\mathrm{~g})$, that $(V, W) /(\phi, \psi)(S, T)$ is torsion-free. Therefore $\left(^{* *}\right)$ 
implies that $v-\phi_{1}-s_{1} \phi_{2} s_{2} \in \phi S$. But $\phi S \subseteq X$, and therefore we have again $v \in X$. Thus $(X, Y)$ is torsion-closed in $(V, W)$ as required. Finally we remove the assumption that $(V, W)$ is torsion-free. Let $(\mu, v)$ be the natural homomorphisms of $(V, W)$ onto $(V, W) / t(V, W)$ and let $\left(\mu_{i}, v_{i}\right), i=1,2$, be endomorphisms of $(S, T)$ at least one of which is an automorphism. Then

$$
\sum_{i=1}^{2}\left((\mu, v)\left(\phi_{i}, \psi_{i}\right)\right)\left(\mu_{i}, v_{i}\right)=(\mu, v) \sum_{i=1}^{2}\left(\phi_{i}, \psi_{i}\right)\left(\mu_{i}, v_{i}\right)
$$

is a q.s. monomorphism by 5.4. Hence by the case of a torsion-free system $\sum_{i=1}^{2}\left(\left(\phi_{i}, \psi_{i}\right)(S, T)+t(V, W)\right) / t(V, W)$ is a q.s. direct sum. This fact and 5.3 yield, as in the proof of 4.2 , the desired conclusion.

5.10. Lemma. Let $\left(X_{i}, Y_{i}\right), i=1,2$, be torsion-free systems of rank 1 of different isomorphism types which are q.s. subsystems of a system $(V, W)$. Suppose in addition that at least one of these subsystems is finite-dimensional or that $\bigcup_{i=1}^{2}\left\{\theta \in \Theta \mid H_{\theta}^{i}=\infty\right\}=\Theta$, where $H^{i} \in \mathscr{H}\left(X_{i}, Y_{i}\right)$. Then $\left(X_{1}, Y_{1}\right)+\left(X_{2}, Y_{2}\right)$ is a direct sum which is q.s. in $(V, W)$.

Proof. Referring to the partial order introduced after 3.5, we may assume, say, that

$$
\mathscr{H}\left(X_{2}, Y_{2}\right) \$ \mathscr{H}\left(X_{1}, Y_{1}\right) .
$$

As in the proof of the previous lemma, if $\left(X_{1}, Y_{1}\right) \cap\left(X_{2}, Y_{2}\right) \neq(0,0)$, then there exists a projection $(\pi, \rho)$ of $\left(X_{1}, Y_{1}\right)+\left(X_{2}, Y_{2}\right)$ onto $\left(X_{1}, Y_{1}\right)$. From (*) it follows that the restriction of $(\pi, \rho)$ to $\left(X_{2}, Y_{2}\right)$ is not a monomorphism. Hence, by 3.1, this restriction vanishes; which shows that $(X, Y)=\left(X_{1}, Y_{1}\right)+\left(X_{2}, Y_{2}\right)$ is a direct sum. We omit the verification that $(X, Y)$ is q.s. in case both systems are of finite dimension since this result was proved in [1]. In case that at least one of the systems is of infinite dimension it suffices by the argument of the preceding lemma to show that, under the additional assumption that $(V, W)$ is torsion-free, $(X, Y)$ is torsion-closed in $(V, W)$. Let $v \in V, \theta \in \Theta$ be such that $B_{\theta} v=y_{1}+y_{2}, y_{i} \in Y_{i}$. If $\left(X_{1}, Y_{1}\right)$ say is finite-dimensional, it is algebraically compact, and there exists a projection $(\pi, \rho)$ of $(V, W)$ onto $\left(X_{1}, Y_{1}\right)$. Again the restriction of $(\pi, \rho)$ to $\left(X_{2}, Y_{2}\right)$ must vanish. Therefore $B_{\theta}(v-\pi v)=B_{\theta} v-\rho\left(y_{1}+y_{2}\right)=B_{\theta} v-y_{1}=y_{2} \in Y_{2}$. Since $\left(X_{2}, Y_{2}\right)$ is torsion-closed in $(V, W)$, it follows that $v-\pi v \in X_{2}$, or $v \in X$. If both systems are of infinite dimension, we have say $H_{\theta}^{1}=\infty$. Then $H\left(y_{1}\right)_{\theta}=\infty$ and there exists an element $x_{1} \in X_{1}$ such that $B_{\theta} x_{1}=y_{1}$. Then $B_{\theta}\left(v-x_{1}\right)=y_{2}$, and we have again $v \in X$.

We remark that in case $\left(X_{i}, Y_{i}\right)$ are both of infinite dimension and there exists a $\theta$ in $\Theta$ with $H_{\theta}^{i}<\infty, i=1,2$, the $\operatorname{sum}(X, Y)$ is not necessarily q.s.: By 3.4 there exist elements $y_{i}$ in $Y_{i}$ such that $H\left(y_{i}\right)_{\theta}=0, i=1,2$. Define a system $(V, W)$ by stipulating that $V=X_{1}+X_{2}+[v], W=Y_{1}+Y_{2}+[w], v, w \neq 0,\left(X_{i}, Y_{i}\right)$ are 
subsystems of $(V, W), B_{\theta} v=y_{1}+y_{2}$ and for some $\eta \neq \theta, B_{\eta} v=w$. Then it is easily verified that $\left(X_{i}, Y_{i}\right)$ are torsion-closed in $(V, W)$, hence q.s. On the other hand $(V, W)$ is torsion-free and $(X, Y)$ is not torsion-closed.

From 4.2, 5.5, 5.9, 5.10 and the result of [1] that the family of q.s.irr. torsion types satisfies the conditions of 4.2 , we obtain:

5.11. THEOREM. Theorem 4.2 remains valid if the conditions on the family of nonisomorphic systems $\left\{\left(S_{j}, T_{j}\right)\right\}$ are replaced by the requirements:

(a) Each $\left(S_{j}, T_{j}\right)$ is either a q.s.irr. torsion system or a torsion-free system of rank 1.

(b) Any two infinite-dimensional torsion-free systems of rank 1 in the family satisfy the condition of 5.10 .

The discussion in $\$ 4$ of the relation $\prec$ between elements and subsets of $D(S, T ; V, W)$ can be completed somewhat in case that $(S, T)$ is torsion-free of rank 1. For such $(S, T)$ this relation satisfies the exchange postulate. As we noted in $\S 4$, it suffices to verify that $\left(\phi_{1}, \psi\right) \prec\left\{\left(\phi, \psi_{1}\right)\right\}$ and $(\phi, \psi)$ not $\prec \varnothing$ imply that $\left(\phi_{1}, \psi_{1}\right) \prec\{(\phi, \psi)\}$. By the results of [1] and 5.4, one has to consider only the case that $(S, T)$ is infinite-dimensional and $(V, W)$ is torsion-free. The verification is then immediate upon inspecting the proof of 5.9. However, as our next result shows, the relation $\prec$ is transitive, and therefore a dependence relation, only for an exceptional class of torsion-free systems of rank 1.

Relative to a given basis $A, B$ of $\mathscr{T}$ we denote by $\mathscr{R}^{\theta}$ the isomorphism type of the torsion free systems of rank 1 with height functions which are finite at the single point $\theta$.

5.12. THEOREM. Let $(S, T)$ be a torsion-free system of rank 1 . Then $D(S, T ; V, W)$ is closed under addition for every system $(V, W)$ if and only if $(S, T)$ is of one of the following types: $\mathrm{III}_{m}, m \geqq-1, \mathscr{R}$ or $\mathscr{R}^{\theta}, \theta \in \Theta$.

Proof. Note that according to 4.4 these are the cases in which $D(S, T ; V, W)$ is always a submodule of $\operatorname{Hom}(S, T ; V, W)$. Corollary 5.4 reduces the question to the case that $(V, W)$ is torsion-free. As we mentioned in $\$ 4$, the result for the types III $_{m}$ was proved in [1]. If $(S, T)$ is of type $\mathscr{R}$ and $(V, W)$ is torsion-free, then $D(S, T ; V, W)$ consists of the zero homomorphism only. Every other homomorphism $(\phi, \psi)$ is a monomorphism by 2.2. If $B_{\eta} v \in \psi T$, then since $(S, T)$ is divisible, there exists an element $s$ in $S$ such that $B_{\eta} \phi s=B_{\eta} v$. Since $(V, W)$ is torsion-free, we have $v=\phi s \in \phi S$. Thus $(\phi, \psi)(S, T)$ is torsion-closed and hence q.s. in $(V, W)$. Choose now as a system of type $\mathscr{R}^{\theta}$ the system $(S, T)=(S, T)_{A, B}^{H}$, where

$$
H_{\eta}= \begin{cases}0 & \text { if } \eta=\theta \\ \infty & \text { otherwise }\end{cases}
$$


Let $\left(\phi_{i}, \psi_{i}\right), i=1,2$, belong to $D(S, T ; V, W)$, where $(V, W)$ is torsion-free. We assume that $\left(\phi_{1}, \psi_{1}\right),\left(\phi_{2}, \psi_{2}\right)$ and $\left(\phi_{1}, \psi_{1}\right)+\left(\phi_{2}, \psi_{2}\right)$ are monomorphisms, since otherwise at least one of them vanishes and there is nothing to prove. From 5.6 it follows that there exist $v_{i} \in V, \eta_{i} \in \Theta$ such that $B_{\eta_{i}} v_{i}=\psi_{i} t_{i}, t_{i} \in T, v_{i} \notin \phi_{i} S$. By the argument used for type $\mathscr{R}$, we must have $\eta_{1}=\eta_{2}=\theta$ and

$$
H\left(t_{1}\right)_{\theta}=H\left(t_{2}\right)_{\theta}=0 .
$$

As in the proof of 5.9, there exist $s_{i} \in S$ such that $t_{i}=B_{\theta} s_{i}+\alpha_{i} \cdot 1(1 \in T)$. Here $\alpha_{1}, \alpha_{2} \neq 0$ because of $\left({ }^{*}\right)$. Define

$$
v=\frac{v_{1}}{\alpha_{1}}+\frac{v_{2}}{\alpha_{2}}-\frac{\phi_{1} s_{1}}{\alpha_{1}}-\frac{\phi_{2} s_{2}}{\alpha_{2}} .
$$

Then $B_{\theta} v=\left(\psi_{1}+\psi_{2}\right) 1$. Since $\left(\phi_{1}, \psi_{1}\right)+\left(\phi_{2}, \psi_{2}\right)$ is a monomorphism and $H(1)_{\theta}=H_{\theta}=0, v \notin\left(\phi_{1}+\phi_{2}\right) S$. Thus $\left(\left(\phi_{1}, \psi_{1}\right)+\left(\phi_{2}, \psi_{2}\right)\right)(S, T)$ is not torsionclosed in $(V, W)$ and $\left(\phi_{1}, \psi_{1}\right)+\left(\phi_{2}, \psi_{2}\right) \in D(S, T ; V, W)$. If $(S, T)$ is not of one of the types mentioned in the theorem and is nonsingular, then using 3.6, 3.7 one constructs two endomorphisms of $(S, T)$ which are not automorphisms but the sum of which is the identity. Thus $D(S, T ; S, T)$ is not closed under addition. If $(S, T)$ is singular of infinite dimension, let $\theta_{1} \neq \theta_{2}$ be elements of $\Theta$ and let $t_{i} \in T$ be such that $H\left(t_{i}\right)_{\theta_{i}}=0, i=1,2$. Define a system $(V, W)$ as follows:

$$
\begin{aligned}
& V=X_{1}+X_{2}+\left[v_{1}\right]+\left[v_{2}\right], \\
& W=Y_{1}+Y_{2}+\left[w_{1}\right]+\left[w_{2}\right],
\end{aligned}
$$

where $\left(X_{i}, Y_{i}\right), i=1,2$, are subsystems of $(V, W)$ isomorphic to $(S, T)$ under the isomorphisms $\left(\phi_{i}, \psi_{i}\right) ; v_{1}, v_{2}, w_{1}, w_{2} \neq 0 ; B_{\theta_{1}} v_{1}=\psi_{1} t_{1}, B_{\theta_{2}} v_{1}=w_{1}, B_{\theta_{1}} v_{2}=w_{2}$, $B_{\theta_{2}} v_{2}=\psi_{2} t_{2}$. It is evident that $(V, W) /\left(X_{i}, Y_{i}\right)$ are not torsion-free and that $\left(\phi_{1}, \psi_{1}\right)+\left(\phi_{2}, \psi_{2}\right)$ is a monomorphism. One verifies that

$$
(V, W) /\left(\left(\phi_{1}, \psi_{1}\right)+\left(\phi_{2}, \psi_{2}\right)\right)(S, T)
$$

is torsion-free (hence so is $(V, W)$ ). Thus $\left(\phi_{i}, \psi_{i}\right) \in D(S, T ; V, W)$ while their sum is a q.s. monomorphism.

Our concluding result shows how in a special case the invariant $\operatorname{Hom}(S, T ; V, W) / D(S, T ; V, W)$ determines the structure of the system.

5.13. THEOREM. Let $(S, T)$ be a singular torsion-free system of rank 1 , and let $(V, W)$ be a direct sum of subsystems isomorphic to $(S, T)$. Then $D(S, T ; V, W)$ consists of the zero homomorphism only. We have $(V, W)=\Sigma+\left(\phi_{i}, \psi_{i}\right)(S, T)$ with $\left(\phi_{i}, \psi_{i}\right)$ monomorphisms if and only if $\left\{\left(\phi_{i}, \psi_{i}\right)\right\}$ is a basis of $\operatorname{Hom}(S, T ; V, W)$ considered as a vector space over the field of complex numbers. Hence the cardinal number of summands in every such decomposition is the dimension of the vector space $\operatorname{Hom}(S, T ; V, W)$. 
Proof. Let $(V, W)=\Sigma+\left(X_{i}, Y_{i}\right)$ be a decomposition of $(V, W)$ into subsystems isomorphic to $(S, T)$, and let $\left(\pi_{i}, \rho_{i}\right)$ be the associated projections. Let $(\phi, \psi)$ be a nonzero element of $\operatorname{Hom}(S, T ; V, W)$. According to $3.1,(\phi, \psi)$ is a monomorphism. Let $t$ be a nonzero element of $T$. Then $K=\left\{i \mid \rho_{i} \psi t \neq 0\right\}$ is a finite nonvoid set, and $\psi t \in \sum_{i \in K} Y_{i}$ or $(\varnothing,\{\psi t\}) \subseteq \sum_{i \in K}\left(X_{i}, Y_{i}\right)$. Since $(\phi, \psi)$ is a monomorphism, $(\phi, \psi)(S, T)$ is torsion-free of rank 1 , and hence $(\phi, \psi)(S, T)=\mathrm{cl}_{(\phi, \psi)(S, T)}(\varnothing,\{\psi t\})$. According to 2.1(e), 2.1(a) and 2.1(g) we have

$$
\begin{aligned}
(\phi, \psi)(S, T) & =\mathrm{cl}_{(\phi, \psi)(s, T)}(\varnothing,\{\psi t\}) \subseteq \mathrm{cl}_{(V, W)}(\varnothing,\{\psi t\}) \subseteq \mathrm{cl}_{(V, W)} \sum_{i \in K}\left(X_{i}, Y_{i}\right) \\
& =\sum_{i \in \mathbf{K}}\left(X_{i}, Y_{i}\right) .
\end{aligned}
$$

Hence $(\phi, \psi)=\sum_{i \in I}\left(\pi_{i}, \rho_{i}\right)(\phi, \psi)$. By the definition of $K$ we have $\left(\pi_{i}, \rho_{i}\right)(\phi, \psi) \neq(0,0)$ for $i \in K$. It follows that if $\left(\sigma_{i}, \tau_{i}\right)$ denotes an isomorphism of $(S, T)$ onto $\left(X_{i}, Y_{i}\right)$, then $\left(\sigma_{i}, \tau_{i}\right)^{-1}\left(\pi_{i}, \rho_{i}\right)(\phi, \psi)$ is a nonzero endomorphism of $(S, T)$. By 3.7 this endomorphism is of the form $\alpha_{i}(1,1), \alpha_{i}$ a nonzero complex number. Therefore $(\phi, \psi)=\sum_{i \in K}\left(\sigma_{i}, \tau_{i}\right) \alpha_{i}$, and 4.1 shows that $(\phi, \psi)$ is q.s. This proves the first statement of the theorem. The necessity part of the second statement follows immediately from the first statement, 5.11 and 3.7. There also show that if $\left\{\left(\phi_{i}, \psi_{i}\right)\right\}$ is a basis of $\operatorname{Hom}(S, T ; V, W)$, then $\Sigma\left(\phi_{i}, \psi_{i}\right)(S, T)$ is a direct sum. That it exhausts $(V, W)$ follows from the fact that for every $j,\left(\sigma_{j}, \tau_{j}\right)$ is a finite linear combination with complex coefficients of elements of the basis; hence $\left(X_{j}, Y_{j}\right)$ is contained in $\Sigma\left(\phi_{i}, \psi_{i}\right)(S, T)$. The last statement of the theorem is obvious.

\section{REFERENCES}

1. N. Aronszajn and U. Fixman, Algebraic spectral theory (to appear).

2. R. Baer, Abelian groups without elements of finite order, Duke Math. J. 3 (1937), 68-122.

3. R. A. Beaumont and H. S. Zuckerman, A characterization of the subgroups of the additive rationals, Pacific J. Math. 1 (1951), 169-177.

4. L. Fuchs, Abelian groups, Publishing House of the Hungarian Academy of Sciences, Budapest, 1958.

5. F. R. Gantmacher, Applications of the theory of matrices (English transl.), Interscience, New York, 1959.

6. I. Kaplansky, Infinite abelian groups, Univ. of Michigan Press, Ann Arbor, Mich., 1954.

7. S. MacLane, A lattice formulation for transcendence degrees and p-bases, Duke Math. J. 4 (1938), 455-468.

YALE UNIVERSTTY,

New Haven, Connecticut

STANFORD UNIVERSITY,

Stanford, California 